\title{
GALAXY EMISSION LINE CLASSIFICATION USING THREE-DIMENSIONAL LINE RATIO DIAGRAMS
}

\author{
Frédéric P. A. Vogt ${ }^{1,2}$, Michael A. Dopita ${ }^{1,3,4}$, Lisa J. Kewley ${ }^{1,4}$, Ralph S. Sutherland ${ }^{1}$, \\ Julia Scharwächter ${ }^{5}$, Hassan M. Basurah ${ }^{3}$, AlaA Ali ${ }^{3,6}$, and Morsi A. Amer ${ }^{3,6}$ \\ ${ }^{1}$ Research School of Astronomy and Astrophysics, Australian National University, Canberra, ACT 2611, Australia; frederic.vogt@anu.edu.au \\ ${ }^{2}$ Department of Physics and Astronomy, Johns Hopkins University, 3400 N. Charles Street, Baltimore, MD 21218, USA \\ ${ }^{3}$ Astronomy Department, King Abdulaziz University, P.O. Box 80203, Jeddah, Saudi Arabia \\ ${ }^{4}$ Institute for Astronomy, University of Hawaii, 2680 Woodlawn Drive, Honolulu, HI 96822, USA \\ ${ }^{5}$ Observatoire de Paris, LERMA (CNRS: UMR8112), 61 Av. de l'Observatoire, F-75014 Paris, France \\ ${ }^{6}$ Astronomy Department, Faculty of Science, Cairo University, Egypt \\ Received 2013 December 11; accepted 2014 June 12; published 2014 September 16
}

\begin{abstract}
Two-dimensional (2D) line ratio diagnostic diagrams have become a key tool in understanding the excitation mechanisms of galaxies. The curves used to separate the different regions-H II-like or excited by an active galactic nucleus (AGN) - have been refined over time but the core technique has not evolved significantly. However, the classification of galaxies based on their emission line ratios really is a multi-dimensional problem. Here we exploit recent software developments to explore the potential of three-dimensional (3D) line ratio diagnostic diagrams. We introduce the $\mathcal{Z Q E}$ diagrams, which are a specific set of 3D diagrams that separate the oxygen abundance and the ionization parameter of $\mathrm{H}$ II region-like spectra and also enable us to probe the excitation mechanism of the gas. By examining these new 3D spaces interactively, we define the $\mathcal{Z E}$ diagnostics, a new set of 2D diagnostics that can provide the metallicity of objects excited by hot young stars and that cleanly separate $\mathrm{H}$ II region-like objects from the different classes of AGNs. We show that these $\mathcal{Z E}$ diagnostics are consistent with the key $\log [\mathrm{N}$ II $] / \mathrm{H} \alpha$ versus $\log [\mathrm{O}$ III $] / \mathrm{H} \beta$ diagnostic currently used by the community. They also have the advantage of attaching a probability that a given object belongs to one class or the other. Finally, we discuss briefly why $\mathcal{Z} \mathcal{Q E}$ diagrams can provide a new way to differentiate and study the different classes of AGNs in anticipation of a dedicated follow-up study.
\end{abstract}

Key words: galaxies: abundances - galaxies: general - galaxies: Seyfert - galaxies: starburst - H II regions ISM: lines and bands

Online-only material: color figures, tar.gz files

\section{INTRODUCTION}

The use of specific line ratios to distinguish line emission regions depending on their gas excitation mechanism was pioneered by Baldwin et al. (1981) and extended by Veilleux \& Osterbrock (1987). The line ratios most frequently used, specifically designed to be insensitive to reddening, are:

1. $\log [\mathrm{N}$ II $] / \mathrm{H} \alpha$ versus $\log [\mathrm{O} I I I] / \mathrm{H} \beta$,

2. $\log [\mathrm{S} \mathrm{II}] / \mathrm{H} \alpha$ versus $\log [\mathrm{O} \mathrm{III}] / \mathrm{H} \beta$, and

3. $\log [\mathrm{O} \mathrm{I}] / \mathrm{H} \alpha$ versus $\log \left[\mathrm{O}_{\mathrm{III}}\right] / \mathrm{H} \beta$.

Theoretical progress has since allowed the placement of different diagnostic lines separating the different excitation mechanisms in these diagrams: regions photoionized by hot stars giving H II-like spectra and regions excited by an active galactic nucleus (AGN), either Seyferts or the low ionization nuclear emission-line regions (LINERs). Currently, the maximum starburst lines from Kewley et al. (2001a, 2001b), the empirical starburst line from Kauffmann et al. (2003a), and the LINER-Seyfert lines from Kewley et al. (2006) are commonly used. Other, similar diagnostics include Heckman (1980), Osterbrock \& Pogge (1985), Veilleux \& Osterbrock (1987), Tresse et al. (1996), Ho et al. (1997), Dopita et al. (2000), and Stasińska et al. (2006).

The maximum starburst lines as defined by Kewley et al. (2001a) are based on theoretical modeling of starburst galaxies. Specifically, the wrap-round of theoretical model grids inside these optical line ratio diagnostic diagrams justifies the definition of a theoretical upper bound of emission line ratios from gas photoionized by hot young stars. Kauffmann et al. (2003a) used the large number statistics of the Sloan Digital
Sky Survey (SDSS; York et al. 2000) to set an observational lower bound to the maximum starburst line in the $\log [\mathrm{N}$ II] $/ \mathrm{H} \alpha$ versus $\log \left[\mathrm{O}_{\mathrm{III}}\right] / \mathrm{H} \beta$ diagram. The region between these two starburst lines is known as the "composite" region. Recently, several objects in the composite region have been recognized as being (at least in part) excited by shocks (Farage et al. 2010; Rich et al. 2011, 2014), although this does not rule out a mixed excitation mechanism (starburst+AGN) for other composite objects (e.g., Scharwächter et al. 2011; Davies et al. 2014; Dopita et al. 2014b). Kewley et al. (2006) also exploited the large number statistics from SDSS to define the separation lines between the LINER and the Seyfert branches on the AGN side of the $\log [\mathrm{S} \mathrm{II}] / \mathrm{H} \alpha$ versus $\log [\mathrm{O} I \mathrm{II}] / \mathrm{H} \beta$ and $\log \left[\mathrm{O}_{\mathrm{I}}\right] / \mathrm{H} \alpha$ versus $\log [\mathrm{O}$ III $] / \mathrm{H} \beta$ diagrams.

The classical optical line ratio diagnostic diagrams have proved to be useful and resilient ever since their introduction. Recently, their usage has been extended as new IR surveys of galaxies measure the key line ratios for galaxies at intermediate and high redshifts. The key instruments are MOSFIRE on Keck (McLean et al. 2010), FMOS on Subaru (Kimura et al. 2010), MMIRS on Magellan (McLeod et al. 2004), FLAMINGOS II on Gemini (Eikenberry et al. 2008), and LUCI at the Large Binocular Telescope (Buschkamp et al. 2012). The sensitivity of the optical line ratio diagnostics to metallicity and other factors that could influence the diagnostics at high redshift was investigated by Kewley et al. (2013a) and this insight was applied to actual samples of high-redshift galaxies by Kewley et al. (2013b).

A further stimulus for the use of optical line ratio diagnostic diagrams has been the advent of integral field spectrographs. These instruments provide spectral information for individual 
spectral pixels (commonly referred to as spaxels). With this approach, it is possible to reveal the presence of metallicity gradients across the entire spatial extent of galaxies (Rich et al. 2012), explore the trends in the local excitation pressure (Dopita et al. 2014a), or study the AGN zone of influence (Scharwächter et al. 2011). These analyses, in turn, rely on an accurate classification scheme.

In reality, the full set of line ratios forms a multi-dimensional space, the topology of which needs to be understood before a final classification can be set. Progress toward this goal can be made by looking at alternative line ratio diagrams. For the case of H II regions, Dopita et al. (2013) made a comprehensive study of the utility of alternative diagnostic diagrams, discussing previously used ones, as well as introducing some new ones. Few of these diagrams separate the AGN branch from the stellar excited objects as well as the traditional $\log \left[\mathrm{N}_{\mathrm{II}}\right] / \mathrm{H} \alpha$ versus $\log [\mathrm{O}$ III $] / \mathrm{H} \beta$ diagram. Notable exceptions are provided by the $\log [\mathrm{N}$ II $] / \mathrm{H} \alpha$ versus $\log [\mathrm{O} \mathrm{III}] /[\mathrm{O} \mathrm{II}]$ diagram and the $\log [\mathrm{N}$ II $] /$ [O II $]$ versus $\log \left[\mathrm{O}_{\mathrm{III}}\right] / \mathrm{H} \beta$ diagram.

In this paper, we revisit the concept of the optical line ratio diagram itself, and introduce new three-dimensional (3D) line ratio diagrams. These diagrams, combining three different and complimentary line ratios, are a first step toward a better understanding of the distribution of galaxies in their multidimensional line ratio space. This article is organized as follows. We first describe the observational data sets that we employ in our analysis in Section 2, and the theoretical models we use in Section 3. We introduce the new 3D line ratio diagrams derived from these data sets in Section 4. In Section 5, we use specific 3D line ratio diagrams to generate a new and consistent set of line ratio diagnostics to separate $\mathrm{H}$ II-like galaxies from the AGNlike objects. In Section 6, we compare these new diagnostics with the standard optical line ratio diagnostic diagrams, and discuss their compatibility with intermediate and high redshift spectroscopic observations. Finally, we highlight the potential of these new 3D line ratio diagrams to investigate the different AGN families in Section 7, and summarize our conclusions in Section 8 .

\section{OBSERVATIONAL DATA SETS}

\subsection{SDSS Galaxies}

We construct our sample of emission line galaxies from the Sloan Digital Sky Survey (SDSS) data release (DR) 8 (Aihara et al. 2011a, 2011b; Eisenstein et al. 2011). Specifically, we exploit the "galSpec" Value Added Catalog from the Max Planck Institute for Astronomy and Johns Hopkins University (MPAJHU) group. The data are in fact identical to those associated with the SDSS DR7 (Abazajian et al. 2009), but was first made accessible via the general SDSS DR in DR8. This data set has been freely accessible since the SDSS DR4 (AdelmanMcCarthy et al. 2006), and the associated fitting procedures for the stellar continuum and emission lines are described in detail in Kauffmann et al. (2003b), Brinchmann et al. (2004), and Tremonti et al. (2004). Each spectrum is corrected for the foreground Galactic extinction using the O'Donnell (1994) extinction curve. The stellar continuum is fitted with a linear combination of 10 single-age stellar population models based on a new version of the GALEXEV code of Bruzual \& Charlot (2003, 2011) plus an additional parameter accounting for internal dust attenuation (Charlot \& Fall 2000). The different emission lines are fitted with single Gaussians. Balmer lines share a unique
Table 1

Corrections Applied to the Errors of Emission Line Fluxes

\begin{tabular}{lc}
\hline \hline Line & Correction \\
\hline$[\mathrm{O}$ II $] \lambda 3726$ & 1.33 \\
{$[\mathrm{O}$ II $] \lambda 3729$} & 1.33 \\
$\mathrm{H} \beta$ & 1.29 \\
{$[\mathrm{O}$ III $] \lambda 5007$} & 1.33 \\
{$[\mathrm{O}$ I $] \lambda 6300$} & 1.02 \\
$\mathrm{H} \alpha$ & 2.06 \\
{$[\mathrm{~N} \mathrm{II}] \lambda 6584$} & 1.44 \\
{$\left[\mathrm{~S} \mathrm{II}^{\mathrm{II}} \lambda 6717\right.$} & 1.36 \\
{$\left[\mathrm{~S} \mathrm{II}_{\mathrm{II}}\right] \lambda 6731$} & 1.36 \\
\hline
\end{tabular}

Note. Although $\mathrm{S} / \mathrm{N}([\mathrm{OI}]$ 26300) is not used in our sample selection, the associated error scaling correction is included here for completeness.

rest-frame velocity and velocity dispersion (accounting for the instrumental resolution), and so do the forbidden lines.

From the 1,843,200 objects provided in DR8, we extract a sub-sample of high-quality spectra with reliable galSpec fit parameters, following the methodology of Kewley et al. (2006). Our detailed selection criteria (including the explicit SDSS keywords expressions) are:

1. an existing galSpec fit (i.e., [PLATEID;FIBERID;MJD] $\neq$ $-1)$

2. the galSpec fit is flagged as "reliable" by the MPA-JHU group (i.e., RELIABLE $=1$ );

3. a reliable redshift measurement (i.e., Z_WARNING $=0$ );

4. a redshift between 0.04 and 0.1 (i.e., $0.04<\mathrm{Z}<0.1$ );

5. a signal-to-noise ratio $(\mathrm{S} / \mathrm{N}) \geqslant 3$ in the following strong lines: [O II] $\lambda 3726,\left[\mathrm{O}_{\mathrm{II}}\right] \lambda 3729, \mathrm{H} \beta,[\mathrm{O} \mathrm{III}] \lambda 5007, \mathrm{H} \alpha$, $[\mathrm{N}$ II] $\lambda 6584,[\mathrm{~S} \mathrm{II}] \lambda 6717$, and [S II] $\lambda 6731$,

6. $\mathrm{S} / \mathrm{N} \geqslant 3$ for the continuum measurement around $\mathrm{H} \beta$; and

7. $\mathrm{H} \alpha / \mathrm{H} \beta_{\text {corr }} \geqslant 2.86$.

The redshift selection is identical to that of Kewley et al. (2006): the lower limit ensures that at least $20 \%$ of the galaxy is covered by the 3 arcsec fiber of the SDSS spectrograph so that the spectra are representative of the global properties of the galaxies (Kewley et al. 2005). The higher redshift bound is designed to ensure the completeness of the LINER class, comparatively dimmer than Seyferts. We calculated the S/N of each emission line from the line flux and its associated error scaled by the amount suggested by Juneau et al. (2014; see Table 1). These correction factors have been obtained by comparing the different duplicate observations in the data set and are lower than the values recommended by the MPAJHU group for their DR4 Value Added Catalog. Following the recommendation of Groves et al. (2012), we also add $0.35 \AA$ to the equivalent width of $\mathrm{H} \beta$ (with $\mathrm{H} \beta_{\text {corr }}$ the corrected line flux), which was found to be underestimated because of an error in the 2008 version of the GALEXEV code (Bruzual \& Charlot 2011). Hence, we require the continuum level around $\mathrm{H} \beta$ to have $\mathrm{S} / \mathrm{N} \geqslant 3$ to ensure a reliable correction. The median correction for our sample is $\sim 6 \%$ of the original $\mathrm{H} \beta$ flux.

We have removed duplicate observations in the sample using our own Python routine. For every galaxy, we look for all other objects located within 3 arcsec (with no restriction on the redshift), and remove them all from our sample except for the one with the largest $\mathrm{S} / \mathrm{N}(\mathrm{H} \alpha)$. Our final sample is composed of 105,070 galaxies. 


\subsubsection{Extragalactic Reddening Correction}

We correct the emission line fluxes for extragalactic reddening based on the Balmer decrement $R_{\alpha \beta}$, using the extinction law from Fischera \& Dopita (2005) for $R_{V}^{A}=4.5$ (and $A_{V}=1$ ). This extinction law is very close to that of Calzetti et al. (2000) for starburst galaxies, and Wijesinghe et al. (2011) have shown that it provides very good agreement between different star-formation rate (SFR) indicators ([O II], H $\alpha$, near-UV, far-UV) for the GAMA galaxies (Driver et al. 2009). Specifically, we follow the procedure described in detail in Appendix A of Vogt et al. (2013), with:

$$
\begin{aligned}
\frac{E_{\lambda-V}}{E_{B-V}}= & -4.61777+1.41612 \cdot \lambda^{-1}+1.52077 \cdot \lambda^{-2} \\
& -0.63269 \cdot \lambda^{-3}+0.07386 \cdot \lambda^{-4}
\end{aligned}
$$

where $\lambda$ is in $\mu \mathrm{m}$, and the actual reddening correction is given by:

$$
F_{\lambda, 0}=F_{\lambda} \cdot\left(\frac{F_{\mathrm{H} \alpha} / F_{\mathrm{H} \beta}}{R_{\alpha \beta}}\right)^{-\frac{\frac{E_{\lambda-V}}{E_{B-V}}+R_{V}^{A}}{E_{H \alpha-V}}-\frac{E_{H \beta-V}}{E_{B-V}}}
$$

with $F_{\lambda, 0}$ the intrinsic emission line flux, $F_{\lambda}$ the measured emission line flux, $\lambda$ the rest-frame emission line wavelength, and in our case, $R_{V}^{A}=4.5$.

We adopt an intrinsic Balmer ratio $R_{\alpha \beta}=2.86$ corresponding to Case B recombination for every object in our sample, irrespective of their classification. This value is appropriate for star-forming galaxies, but the presence of an AGN can result in a higher intrinsic Balmer ratio (i.e., $R_{\alpha \beta} \cong 3.1$; e.g., Osterbrock 1989; Kewley et al. 2006). However, it is unclear what intrinsic ratio should be applied for "composite" objects possibly containing a mix of star formation and AGNs. Hence, we use an intrinsic Balmer ratio of 2.86 to ensure a uniform sample without artificial separation. For consistency, we will indicate visually in all line ratio diagrams throughout this article the spatial displacement $\zeta$ associated with an intrinsic Balmer decrement $R_{\alpha \beta}=3.1$ instead of 2.86. Analytically, for an observed line ratio $F_{\lambda_{1}} / F_{\lambda_{2}}$, we can write using Equation (2):

$$
\frac{F_{\lambda_{1}, 0}}{F_{\lambda_{2}, 0}}=\frac{F_{\lambda_{1}}}{F_{\lambda_{2}}} \cdot\left(\frac{2.86}{R_{\alpha \beta}}\right)^{\frac{\tau_{\lambda_{2}}-\tau_{\lambda_{1}}}{\tau_{\mathrm{H} \alpha}-\tau_{\mathrm{H} \beta}}} \cdot\left(\frac{F_{\mathrm{H} \alpha} / F_{\mathrm{H} \beta}}{2.86}\right)^{\frac{\tau_{\lambda_{2}}-\tau_{\lambda_{1}}}{\tau_{\mathrm{H} \alpha}-\tau_{\mathrm{H} \beta}}}
$$

where

$$
\tau_{\lambda}=\frac{E_{\lambda-V}}{E_{B-V}}+R_{V}^{A}
$$

so that

$$
\zeta\left(\lambda_{1}, \lambda_{2}\right)=\left(\frac{2.86}{R_{\alpha \beta}}\right)^{\frac{\tau_{\lambda_{2}}-\tau_{1}}{\tau_{\mathrm{H} \alpha}-\tau_{\mathrm{H} \beta}}}
$$

As we will discuss in the next sections, $\zeta$ is small enough so that the choice of $R_{\alpha \beta}$ is not critical to our analysis. Especially, as we will focus on the separation between AGNdominated and star-forming galaxies, the objects located close to or on the classification diagnostic lines (i.e., with very little AGN influence) can be expected to have $R_{\alpha \beta} \cong 2.86$. As mentioned above, we have removed $\sim 200$ galaxies with measured $\mathrm{H} \alpha / \mathrm{H} \beta_{\text {corr }}<2.86$ from our sample, under the assumption that these low ratios are indicative of observational and/or fitting issues.
After correcting the emission line fluxes for extragalactic reddening, our sample contains $88,933(84.6 \%)$ galaxies classified as star-forming, 11,447 (10.9\%) classified as composites, and $4690(4.5 \%)$ classified as AGN-dominated, based solely on their position in the $\log [\mathrm{N}$ II $] / \mathrm{H} \alpha$ versus $\log [\mathrm{O}$ III $] / \mathrm{H} \beta$ diagram.

\subsection{H II Region Spectra}

Since the SDSS spectra represent nuclear spectra of whole galaxies, it is important for classification purposes that we also have a set of well-observed isolated $\mathrm{H}$ II regions covering a wide range of chemical abundances. For this purpose, we adopt the excellent homogeneous data set from van Zee et al. (1998).

This data set is somewhat deficient in the most metal-rich objects, so we have supplemented the van Zee et al. (1998) H II regions with our own data on the H II regions in the Seyfert galaxy NGC 5427. These bright $\mathrm{H}$ II regions are unaffected by the weak Seyfert 2 nucleus, and their abundances range up to three times solar. We refer the reader to Dopita et al. (2014b) for more details on the observations, data reduction, and emission line flux measurements for these H II regions.

\section{THE THEORETICAL H II REGION MODELS}

Throughout this article, we rely on the grids of line intensities for $\mathrm{H}$ II regions derived from the modeling code MAPPINGS $I V$ by Dopita et al. (2013). These grids cover a wide range of abundances $\left(5-0.05 Z_{\odot}\right)$ and ionization parameters $(6.5 \lesssim$ $\log q \lesssim 8.5)$

MAPPINGS IV is the latest evolution of the MAPPINGS code (Dopita et al. 1982; Binette et al. 1982, 1985; Sutherland \& Dopita 1993; Groves et al. 2004; Allen et al. 2008), that (among other updates) can now account for the possible nonMaxwellian energy distribution of electrons in astrophysical plasmas. The idea that the energy distribution of electrons in planetary nebulae and $\mathrm{H}$ II regions may depart from a standard Maxwell-Boltzmann distribution to resemble a $\kappa$ distribution, characterized by a high-energy tail, was recently suggested by Nicholls et al. (2012). The consequences of a $\kappa$ distribution of electron energies on temperature and abundance measurements in $\mathrm{H}$ II regions have been discussed in detail by Nicholls et al. (2013). Dopita et al. (2013) found the effects of a $\kappa$ distribution on strong line intensities to be only minor, so that the choice of $\kappa$ does not significantly affect our analysis. Throughout this paper, we have adopted $\kappa=20$.

\section{CREATING 3D LINE RATIO DIAGRAMS}

There is a priori no reason to restrict line ratio diagrams to two dimensions (2D) other than the evident practicality of visualization. Here, we exploit recent software developments to explore the potential of 3D line ratio diagrams. The basic concept is as follows. As a starting point we use the 2D diagnostics from Dopita et al. (2013) which cleanly separate the ionization parameter, $q$, and the oxygen abundance, $12+\log (\mathrm{O} /$ $\mathrm{H})$. We then couple them with an additional line ratio, chosen specifically to help differentiate Hir-like objects from AGNs. This third ratio ought to be more sensitive to the hardness of the radiation field. In Table 2, we list the different line ratios used for each of the three categories,

1. Category I: abundance sensitive ratios,

2. Category II: $q$-sensitive ratios, and

3. Category III: radiation hardness-sensitive ratios. 
Table 2

Line Ratios and Associated Keys

\begin{tabular}{|c|c|c|c|c|c|}
\hline \multicolumn{2}{|c|}{ Category I } & \multicolumn{2}{|c|}{ Category II } & \multicolumn{2}{|c|}{ Category III } \\
\hline Key & Ratio & Key & Ratio & Key & Ratio \\
\hline $\mathrm{a}$ & $\log \frac{[\mathrm{NII}]}{[\mathrm{OII}]}$ & c & $\log \frac{[\mathrm{OIII}]}{[\mathrm{OII}]}$ & f & $\log \frac{[\mathrm{OIII}]}{\mathrm{H} \beta}$ \\
\hline \multirow[t]{3}{*}{$\mathrm{b}$} & $\log \frac{[\mathrm{NII}]}{[\mathrm{S} \mathrm{II}]}$ & d & $\log \frac{[\mathrm{O} \mathrm{IIII}]}{[\mathrm{S} \mathrm{II}]}$ & $\mathrm{g}$ & $\log \frac{[\mathrm{NII}]}{\mathrm{H} \alpha}$ \\
\hline & & $\mathrm{e}$ & $\log \frac{[\mathrm{OIII}]}{[\mathrm{N} \mathrm{III}]}$ & $\mathrm{h}$ & $\log \frac{[\mathrm{S} \mathrm{II}]}{\mathrm{H} \alpha}$ \\
\hline & & & & $\mathrm{i}$ & $\log \frac{[\mathrm{OI}]}{\mathrm{H} \alpha}$ \\
\hline
\end{tabular}

Note. Throughout this paper and unless noted otherwise, when we refer to specific emission lines we mean $[\mathrm{N}$ II $] \equiv[\mathrm{N} \mathrm{II}] \lambda 6583$, [S II $] \equiv[\mathrm{S}$ II $] \lambda 6717+\lambda 6731$, $\left[\mathrm{O}_{\mathrm{II}}\right] \equiv[\mathrm{O}$ II $] \lambda 3727+\lambda 3729,[\mathrm{O} \mathrm{III}] \equiv[\mathrm{O}$ III $] \lambda 5007$ and $\left[\mathrm{O}_{\mathrm{I}}\right] \equiv\left[\mathrm{O}_{\mathrm{I}}\right] \lambda 6300$.

In practice, of course, the separation is not as clean as implied by this list, since each ratio is in some part sensitive to all three parameters we are trying to dissociate. Nonetheless the exercise remains useful as a means of teasing out these parameters.

We restrict ourselves to ratios involving (usually) intense emission lines commonly observed in both H II regions and AGN-dominated objects. We associate a "key," defined in Table 2, with each ratio to unambiguously identify them throughout this paper.

One example of a $3 \mathrm{D}$ line ratio diagram $(\log [\mathrm{N} \mathrm{II}] /[\mathrm{O}$ II $]$ versus $\log [\mathrm{O} \mathrm{III}] /[\mathrm{S} \mathrm{II}]$ versus $\log [\mathrm{N} \mathrm{II}] / \mathrm{H} \alpha$ ) is shown in Figure 1. An interactive version of Figure 1 can be downloaded as a supplementary HTML file, and is also directly accessible online (http://dx.doi.org/10.1088/0004-637X/793/2/127/data). The interactive HTML file (created via the Python module Mayavi2, see Ramachandran \& Varoquaux 2011) allows the reader to freely rotate and zoom in or out of the 3D diagram, as well as to toggle on or off the different datasets. Figure 1 is also 3D printable using the .STL file provided as a supplementary material (see Appendix B for more details). We refer to these new 3D line ratio diagrams as $\mathcal{Z} \mathcal{Q} \mathcal{E}$ diagrams, following the categorization of the line ratios involved. To uniquely identify all possible $\mathcal{Z} \mathcal{Q}$ diagrams, we attach the key of the three line ratios involved (in the order defining a right-handed orthogonal base), in the form of $\mathcal{Z} \mathcal{E}_{\mathrm{x}_{1} \mathrm{x}_{2} \mathrm{x}_{3}}$, where $\mathrm{x}_{1}, \mathrm{x}_{2}$, and $\mathrm{x}_{3}$ correspond to keys of line ratios in Categories I, II, and III defined in Table 2. For example, the $3 \mathrm{D}$ line ratio diagram shown in Figure 1 is $\mathcal{Z} \mathcal{Q E}_{\text {adg }}$.

In this new 3D diagram, the spatial structure of the cloud of points of SDSS galaxies resembles that of a nudibranch. H II-like objects are located on, or close to, the photoionization model grid and can be associated with the sea slug's body. This sequence is clearly separated from the AGN sequence, which extends away from the $\mathrm{H}$ II region model grid (and which can be regarded as the "feelers" of the nudibranch). These AGNdominated regions also display a clear substructure in the spatial density of galaxies, best revealed in the interactive version of Figure 1.

From the line ratios listed in Table 2, it is possible to construct $2 \times 3 \times 4=24$ different $3 \mathrm{D}$ spaces combining one ratio of each category, and we list them all in Table 3 with their $\mathcal{Z} \mathcal{Q E}$ denomination.

\subsection{Exploiting $\mathcal{Z} \mathcal{Q} \mathcal{E}$ Diagrams}

One of the key advantages of $\mathcal{Z} \mathcal{Q} \mathcal{E}$ diagrams is the ability to inspect them interactively (in a similar manner to the interactive counterpart of Figure 1). Following this approach, it is possible to identify new points of view of interest on the multidimensional space of galaxy line ratios. Working interactively
Table 3

All Possible $\mathcal{Z} \mathcal{Q} \mathcal{E}$ Diagrams Combining One Line Ratio from Each of the Categories I, II, and III Defined in Table 2, and the Existence of an Associated $\mathcal{Z} \mathcal{E}$ Diagnostic

\begin{tabular}{|c|c|c|c|c|}
\hline Name & Ratio 1 & Ratio 2 & Ratio 3 & $\mathcal{Z E}$ Diagnostic? \\
\hline $\mathcal{Z} \mathcal{Q} \mathcal{E}_{\text {acf }}$ & $\log \frac{[\mathrm{N} \mathrm{II}]}{[\mathrm{OII}]}$ & $\log \frac{[\mathrm{OIII}]}{[\mathrm{OII}]}$ & $\log \frac{[\mathrm{OIII}]}{[\mathrm{H} \beta]}$ & Yes \\
\hline $\mathcal{Z} \mathcal{Q} \mathcal{E}_{\text {acg }}$ & $\log \frac{[\mathrm{N} \mathrm{II}]}{[\mathrm{OII}]}$ & $\log \frac{[\mathrm{OIII}]}{[\mathrm{OII}]}$ & $\log \frac{[\mathrm{NII}]}{[\mathrm{H} \alpha]}$ & Yes \\
\hline $\mathcal{Z} \mathcal{Q} \mathcal{E}_{\text {ach }}$ & $\log \frac{[\mathrm{N} \mathrm{III}]}{[\mathrm{OII}]}$ & $\log \frac{[\mathrm{OIII}]}{[\mathrm{OII}]}$ & $\log \frac{[\mathrm{S} \mathrm{II}]}{[\mathrm{H} \alpha]}$ & No \\
\hline $\mathcal{Z} \mathcal{Q} \mathcal{E}_{\text {aci }}$ & $\log \frac{[\mathrm{N} \mathrm{II}]}{[\mathrm{OII}]}$ & $\log \frac{[\mathrm{OIII}]}{[\mathrm{OII}]}$ & $\log \frac{[\mathrm{OI}]}{[\mathrm{H} \alpha]}$ & No \\
\hline $\mathcal{Z} \mathcal{Q} \mathcal{E}_{\text {adf }}$ & $\log \frac{[\mathrm{N} \mathrm{III}]}{[\mathrm{OIII}]}$ & $\log \frac{[\mathrm{OIII}]}{[\mathrm{S} \mathrm{II}]}$ & $\log \frac{[\mathrm{OIII}]}{[\mathrm{H} \beta]}$ & No \\
\hline $\mathcal{Z} \mathcal{Q} \mathcal{E}_{\text {adg }}$ & $\log \frac{[\mathrm{N} \mathrm{II}]}{[\mathrm{OII}]}$ & $\log \frac{[\mathrm{OIII}]}{[\mathrm{S} \mathrm{II}]}$ & $\log \frac{[\mathrm{NII}]}{[\mathrm{H} \alpha]}$ & Yes \\
\hline $\mathcal{Z} \mathcal{Q} \mathcal{E}_{\text {adh }}$ & $\log \frac{[\mathrm{N} \mathrm{II}]}{[\mathrm{OII}]}$ & $\log \frac{[\mathrm{OIII}]}{[\mathrm{S} \mathrm{II}]}$ & $\log \frac{[\mathrm{S} \mathrm{II}]}{[\mathrm{H} \alpha]}$ & No \\
\hline $\mathcal{Z} \mathcal{Q} \mathcal{E}_{\text {adi }}$ & $\log \frac{[\mathrm{N} \mathrm{II}]}{[\mathrm{OIII}]}$ & $\log \frac{[\mathrm{OIII}]}{[\mathrm{S} \mathrm{II}]}$ & $\log \frac{[\mathrm{OI}]}{[\mathrm{H} \alpha]}$ & No \\
\hline $\mathcal{Z} \mathcal{Q} \mathcal{E}_{\text {aef }}$ & $\log \frac{[\mathrm{NII}]}{[\mathrm{OII}]}$ & $\log \frac{[\mathrm{OIII}]}{[\mathrm{N} \mathrm{II}]}$ & $\log \frac{[\mathrm{OIII}]}{[\mathrm{H} \beta]}$ & Yes \\
\hline $\mathcal{Z} \mathcal{Q} \mathcal{E}_{\text {aeg }}$ & $\log \frac{[\mathrm{NII}]}{[\mathrm{OIII}]}$ & $\log \frac{[\mathrm{OIII}]}{[\mathrm{NII}]}$ & $\log \frac{[\mathrm{NII}]}{[\mathrm{H} \alpha]}$ & Yes \\
\hline $\mathcal{Z} \mathcal{Q} \mathcal{E}_{\text {aeh }}$ & $\log \frac{[\mathrm{N} \mathrm{II}]}{[\mathrm{OII}]}$ & $\log \frac{[\mathrm{OIII}]}{[\mathrm{NII}]}$ & $\log \frac{[\mathrm{S} \mathrm{II}]}{[\mathrm{H} \alpha]}$ & No \\
\hline $\mathcal{Z} \mathcal{Q} \mathcal{E}_{\text {aei }}$ & $\log \frac{[\mathrm{N} \mathrm{II}]}{[\mathrm{OII}]}$ & $\log \frac{[\mathrm{OIII}]}{[\mathrm{NII}]}$ & $\log \frac{[\mathrm{OI}]}{[\mathrm{H} \alpha]}$ & No \\
\hline $\mathcal{Z} \mathcal{Q} \mathcal{E}_{\text {bcf }}$ & $\log \frac{[\mathrm{NII}]}{[\mathrm{SII}]}$ & $\log \frac{[\mathrm{OIII}]}{[\mathrm{OII}]}$ & $\log \frac{[\mathrm{OIII}]}{[\mathrm{H} \beta]}$ & No \\
\hline $\mathcal{Z} \mathcal{Q} \mathcal{E}_{\mathrm{bcg}}$ & $\log \frac{[\mathrm{N} \mathrm{II}]}{\left[\mathrm{SII}^{2}\right]}$ & $\log \frac{[\mathrm{OIII}]}{[\mathrm{OII}]}$ & $\log \frac{[\mathrm{NII}]}{[\mathrm{H} \alpha]}$ & Yes \\
\hline $\mathcal{Z} \mathcal{Q} \mathcal{E}_{\text {bch }}$ & $\log \frac{[\mathrm{N} \mathrm{II}]}{\left[\mathrm{S} \mathrm{III}^{2}\right.}$ & $\log \frac{[\mathrm{OIII}]}{[\mathrm{OIII}]}$ & $\log \frac{[\mathrm{S} \mathrm{III}]}{[\mathrm{H} \alpha]}$ & Yes \\
\hline $\mathcal{Z} \mathcal{Q} \mathcal{E}_{\text {bci }}$ & $\log \frac{[\mathrm{N} \mathrm{II}]}{\left[\mathrm{S} \mathrm{II}^{2}\right.}$ & $\log \frac{[\mathrm{OIII}]}{[\mathrm{OII}]}$ & $\log \frac{[\mathrm{OI}]}{[\mathrm{H} \alpha]}$ & No \\
\hline $\mathcal{Z} \mathcal{Q} \mathcal{E}_{\text {bdf }}$ & $\log \frac{[\mathrm{N} \mathrm{II}]}{[\mathrm{S} \mathrm{II}]}$ & $\log \frac{[\mathrm{OIII}]}{[\mathrm{S} \mathrm{II}]}$ & $\log \frac{[\mathrm{OIII}]}{[\mathrm{H} \beta]}$ & Yes \\
\hline $\mathcal{Z} \mathcal{Q} \mathcal{E}_{\text {bdg }}$ & $\log \frac{[\mathrm{N} \mathrm{II}]}{[\mathrm{S} \mathrm{II}]}$ & $\log \frac{[\mathrm{OIII}]}{[\mathrm{S} \mathrm{II}]}$ & $\log \frac{[\mathrm{N} \mathrm{II}]}{[\mathrm{H} \alpha]}$ & Yes \\
\hline $\mathcal{Z} \mathcal{Q} \mathcal{E}_{\text {bdh }}$ & $\log \frac{[\mathrm{N} \mathrm{II}]}{[\mathrm{S} \mathrm{II}]}$ & $\log \frac{[\mathrm{OIII}]}{[\mathrm{S} \mathrm{II}]}$ & $\log \frac{[\mathrm{S} \mathrm{II}]}{[\mathrm{H} \alpha]}$ & Yes \\
\hline $\mathcal{Z} \mathcal{Q} \mathcal{E}_{\text {bdi }}$ & $\log \frac{[\mathrm{N} \mathrm{II}]}{[\mathrm{S} \mathrm{II}]}$ & $\log \frac{[\mathrm{OIII}]}{[\mathrm{S} \mathrm{III}]}$ & $\log \frac{[\mathrm{OI}]}{[\mathrm{H} \alpha]}$ & No \\
\hline $\mathcal{Z} \mathcal{Q} \mathcal{E}_{\text {bef }}$ & $\log \frac{[\mathrm{N} \mathrm{II}]}{\left[\mathrm{S} \mathrm{II}^{2}\right.}$ & $\log \frac{[\mathrm{OIII}]}{[\mathrm{NII}]}$ & $\log \frac{[\mathrm{OIII}]}{[\mathrm{H} \beta]}$ & Yes \\
\hline $\mathcal{Z} \mathcal{Q} \mathcal{E}_{\text {beg }}$ & $\log \frac{[\mathrm{N} \mathrm{III}]}{[\mathrm{S} \mathrm{II}]}$ & $\log \frac{[\mathrm{OIIII}]}{[\mathrm{NII}]}$ & $\log \frac{[\mathrm{NII}]}{[\mathrm{H} \alpha]}$ & Yes \\
\hline $\mathcal{Z} \mathcal{Q} \mathcal{E}_{\text {beh }}$ & $\log \frac{[\mathrm{N} \mathrm{II}]}{[\mathrm{S} \mathrm{II}]}$ & $\log \frac{[\mathrm{OIII}]}{[\mathrm{NII}]}$ & $\log \frac{[\mathrm{S} \mathrm{II}]}{[\mathrm{H} \alpha]}$ & Yes \\
\hline $\mathcal{Z} \mathcal{Q} \mathcal{E}_{\text {bei }}$ & $\log \frac{[\mathrm{N} \mathrm{II}]}{[\mathrm{S} \mathrm{II}]}$ & $\log \frac{[\mathrm{OIII}]}{[\mathrm{NII}]}$ & $\log \frac{[\mathrm{OI}]}{[\mathrm{H} \alpha]}$ & No \\
\hline
\end{tabular}

with 3D line ratio diagrams may seem (at first) cumbersome. As we will argue here, it really is not the case anymore. We rely on the Python module Mayavi2 to create our interactive 3D diagrams (Ramachandran \& Varoquaux 2011). We refer the reader to the full package documentation available online. ${ }^{7}$ Mayavi2 is a module dedicated to " $3 \mathrm{D}$ scientific data visualization and plotting in Python." It is in some ways reminiscent of the Matplotlib module dedicated to 2D plotting (Hunter 2007). We stress here that unlike dedicated computer-assisted design software, using Mayavi2 does not require any specific knowledge a priori. The module syntax is relatively intuitive, as illustrated by the basic examples available online. ${ }^{8}$ Similar to other Python modules, Mayavi2 can be integrated seamlessly in any given Python script, and within a few lines, allows the creation of an interactive 3D model, for example, a $\mathcal{Z} \mathcal{Q}$ diagram. We note that in addition to a "cursor-based" approach, the interactive diagrams generated with Mayavi2 can also be manipulated from a Python shell and scripts. Readers with practical questions regarding the implementations of interactive 3D diagrams with Python are welcome to contact us.

At the time of publication of this article, the interactive 3D models generated by Mayavi2 cannot be directly integrated in documents in a portable document format (PDF). While Mayavi2 can save 3D models in various dedicated file formats

\footnotetext{
7 http://docs.enthought.com/mayavi/mayavi/, accessed on 2013 October 29.

8 http://docs.enthought.com/mayavi/mayavi/auto/examples.html
} 

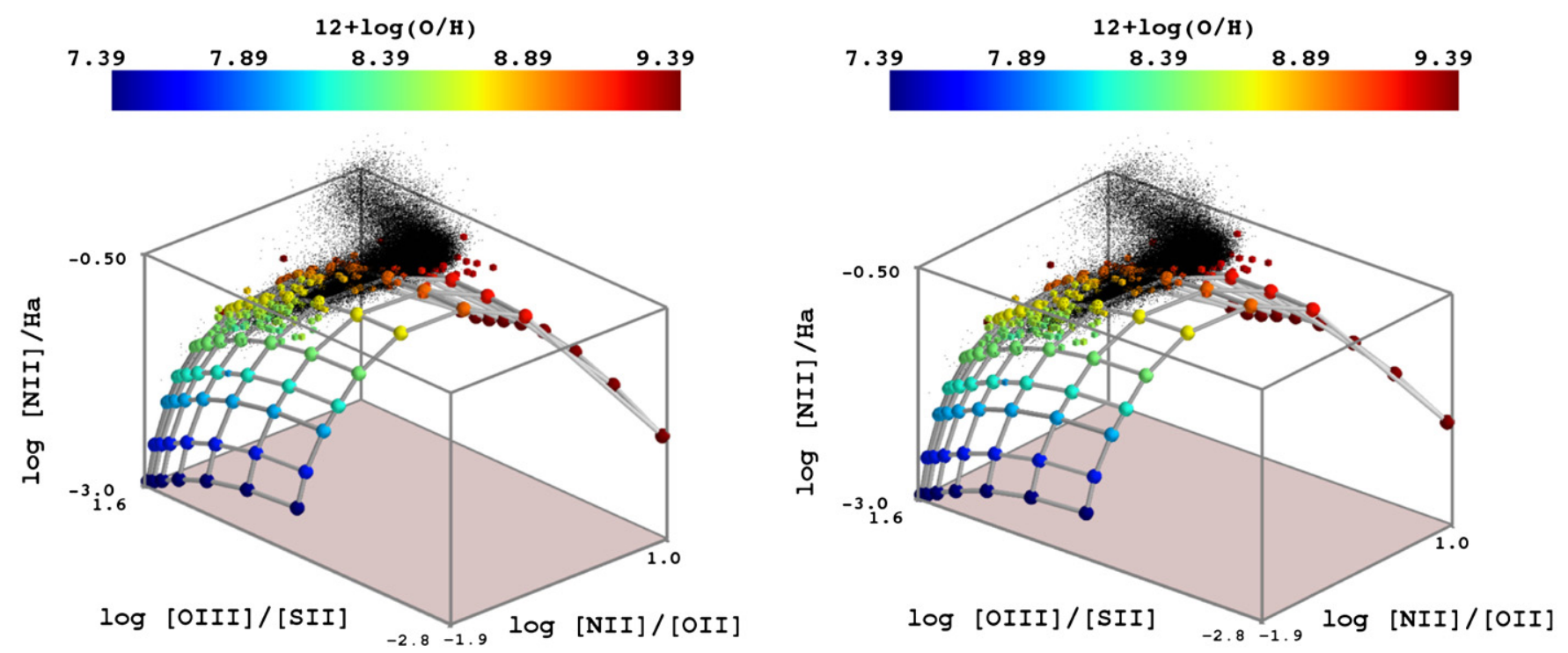

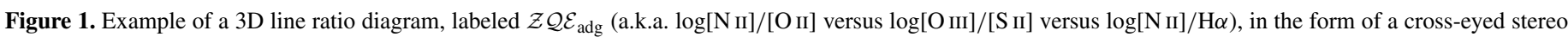

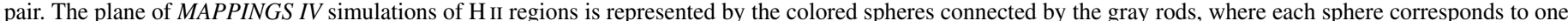

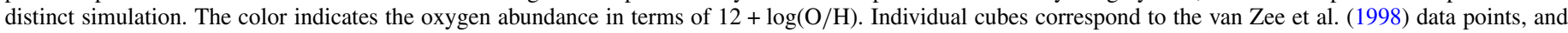

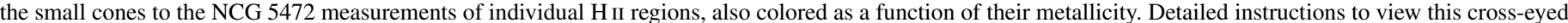

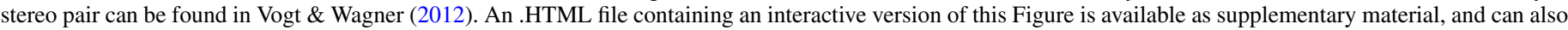
be directly accessed online (http://dx.doi.org/10.1088/0004-637X/793/2/127/data).

(A color version and supplemental data of this figure are available in the online journal.)

(e.g., .VRML, .X3D, .OBJ, .IV), an additional step is required to transform these into the .U3D format, compatible for inclusion in PDF documents. For example, Vogt \& Shingles (2013) relied on the commercial software PDF3DReportGen to transform their VRML file into a .U3D file. Generating interactive .HTML documents is by comparison much more straight-forward. In the present case, we relied on the possibilities offered by HTML5 and $\mathrm{X} 3 \mathrm{DOM}^{9}$ to generate the interactive. $\mathrm{HTML}$ complement to Figure 1. Using simple HTML synthax, a 3D model exported in the .X3D format with Mayavi2 (or any other tool) is directly accessible with many web browsers (Firefox, Safari, Chrome, IE). We refer the interested reader to the X3DOM website for further details regarding the exact list of web browsers compatibility.

\section{FROM 3D $\mathcal{Z} \mathcal{Q E}$ DIAGRAMS TO NEW 2D LINE RATIOS DIAGNOSTICS}

Having introduced the interactive $\mathcal{Z} \mathcal{Q} \mathcal{E}$ 3D line ratio diagrams as a new tool for studying the multi-dimensional galaxy emission line space, we turn our attention to one possible application: the definition of new diagnostic diagrams to separate H II-like and AGN-like objects independently of the standard line ratio diagrams. To that end, we have visually and interactively inspected all $24 \mathcal{Z} \mathcal{Q E}$ diagrams and selected a subsample of 13 in which the starburst sequence and the AGN sequence are best separated. The other 11 diagrams (which do not have a $\mathcal{Z} \mathcal{E}$ diagnostic associated with them in Table 3 ) do not show an evident separation between the AGN and starburst sequences. Hence, these diagrams are less suitable for the kind of analysis presented here. As we discuss in Section 7, these $\mathcal{Z} \mathcal{Q} \mathcal{E}$ diagrams may become of interest in a different type of application, for example, when looking at the inherent structure of the AGN branch in the multi-dimensional line ratio space of galaxy spectra, which will be explored in a separate article.

\footnotetext{
9 http://www.x3dom.org/, accessed on 2014, Aug. 26th
}

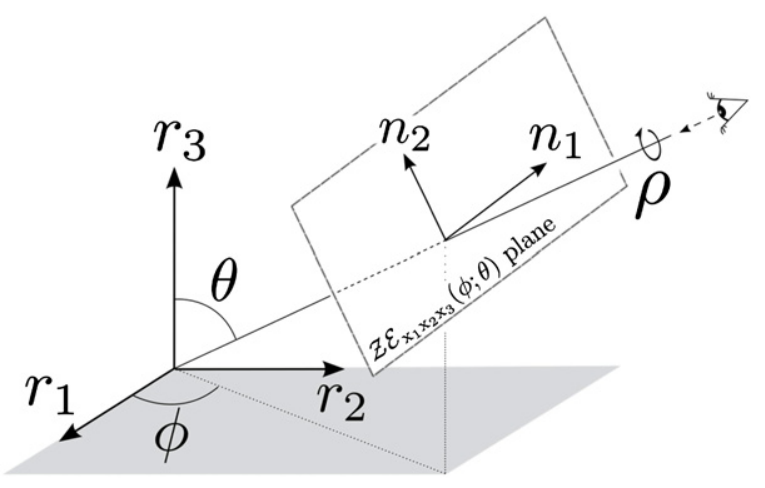

Figure 2. Schematic illustrating the concept of the $\mathcal{Z E}_{\mathrm{x}_{1} \mathrm{x}_{2} \mathrm{x}_{3}}(\phi ; \theta)$ diagram and the associated notation defined in this article.

\subsection{The $\mathcal{Z E}_{\mathrm{X}_{1} \mathrm{X}_{2} \mathrm{x}_{3}}(\phi ; \theta)$ Diagrams}

The original MAPPINGS IV simulation grids created by Dopita et al. (2013) define a set of surfaces in the $\mathcal{Z} \mathcal{Q E}$ line ratio spaces (see Figure 1). For some of the grids, the intrinsic curvature in the third dimension is small, such that it is possible to find a specific point of view from which the grid collapses onto itself, with a thickness $\lesssim 0.3$ dex. By identifying these specific viewpoints, we effectively identify new (composite) 2D line ratio diagrams - the $\mathcal{Z E}_{\mathrm{x}_{1} \mathrm{x}_{2} \mathrm{X}_{3}}(\phi ; \theta)$ diagrams - which rely on the combination of three different line ratios, and in which $\mathrm{H}$ II-like objects are degenerate and constrained to a small region in the diagram.

These new 2D line ratio diagrams are uniquely defined by

1. the three line ratios involved, and

2. the angles $\phi$ and $\theta$ defining the viewing angle in the $\mathcal{Z} \mathcal{Q}$ space defined by the ratios.

Here $\phi$ and $\theta$ are defined following the standard convention for spherical coordinates (see Figure 2). We adopt the convention that the roll angle $\rho=0$ to ensure the uniqueness of each 
diagram. For reasons highlighted below, we refer to these 2D line ratio diagrams constructed from the projection of a $\mathcal{Z} \mathcal{Q}$ space as metallicity-excitation $(\mathcal{Z E})$ diagrams. With this naming convention, we avoid the confusion that may arise through the use of the standard optical line ratio diagnostic diagrams, or with the more recent mass-excitation (MEx) diagram from Juneau et al. $(2011,2014)$.

We introduce the following notation (illustrated in Figure 2), which allows one to uniquely identify any $\mathcal{Z} \mathcal{E}$ diagram (always provided that $\rho=0$ ):

$$
\mathcal{Z E}_{\mathrm{x}_{1} \mathrm{x}_{2} \mathrm{x}_{3}}(\phi ; \theta) \quad \text { with } \quad \phi \in[0,180[; \theta \in[0,180[,
$$

where $\mathrm{x}_{1}, \mathrm{x}_{2}$, and $\mathrm{x}_{3}$ are the three line ratio keys involved (as defined in Table 2). We limit $\phi$ to $180^{\circ}$ to avoid a mirror version of each diagram.

For any given triplet of line ratio values $\left(r_{1} ; r_{2} ; r_{3}\right)$, the $\mathcal{Z E}_{\mathrm{x}_{1} \mathrm{x}_{2} \mathrm{x}_{3}}(\phi ; \theta)$ diagram associates a unique doublet of composite line ratios $\left(n_{1} ; n_{2}\right)$, defined by:

$$
\begin{gathered}
n_{1}=-r_{1} \sin \phi+r_{2} \cos \phi \\
n_{2}=-r_{1} \cos \phi \cos \theta-r_{2} \sin \phi \cos \theta+r_{3} \sin \theta .
\end{gathered}
$$

One should note that because we adopted the convention of $\rho=0$, the composite line ratio $n_{1}$ is simply a combination of the first two ratios, $r_{1}$ and $r_{2}$.

\subsection{The $\mathcal{Z E}_{\mathrm{x}_{1} \mathrm{x}_{2} \mathrm{x}_{3}}\left(\phi^{*} ; \theta^{*}\right)$ Diagnostics}

From the 24 initial $\mathcal{Z} \mathcal{Q E}$ diagrams listed in Table 3, we have identified thirteen for which:

1. we could find a $\mathcal{Z} \mathcal{E}$ plane in which the $\mathrm{H}$ II regions collapse onto a line with a thickness $\lesssim 0.3$ dex, and for which

2. the starburst branch of the SDSS galaxies is well separated from the AGN-like objects.

Hence, we can construct 13 new composite line ratio diagnostic diagrams to classify galaxies as $\mathrm{H}$ II-like or AGN-like. In the next two subsections, we describe in detail how we determine the specific angles $\phi^{*}, \theta^{*}$ and the diagnostic line parameters for each of the $\mathcal{Z} \mathcal{E}$ diagrams.

\subsubsection{Identifying $\phi^{*}$ and $\theta^{*}$ : Manual versus Automated Approach}

We define $\left(\theta^{*} ; \phi^{*}\right)$ the specific values of $\phi$ and $\theta$ which define the $13 \mathcal{Z E}$ diagrams suitable for classifying galaxies as $\mathrm{H}$ IIlike or AGN-like. Each $\mathcal{Z} \mathcal{E}_{\mathrm{x}_{1} \mathrm{x}_{2} \mathrm{x}_{3}}\left(\phi^{*}, \theta^{*}\right)$ diagram is shown in Figures $3-5$. The corresponding $\mathcal{Z} \mathcal{E}_{\mathrm{x}_{1} \mathrm{x}_{2} \mathrm{x}_{3}}\left(\phi^{*} ; \theta^{*}\right)$ denomination is shown in the top left corner of each diagram. For clarity, the $x$ and $y$ axes are labeled with the complete $n_{1}$ and $n_{2}$ composite line ratio equations, derived from Equations (7) and (8). All the parameters of the $13 \mathcal{Z E}$ diagrams are also summarized in Table 4. In each diagram, we show in the top right the median error associated with the SDSS data points given the mix of line ratios involved. Juneau et al. (2014) observed (in a set of duplicate observations extracted from SDSS DR7) that the errors associated with line ratios are comparatively smaller than those associated with individual line fluxes. Hence, our median errors (computed from the individual line errors) reported in the different panels of Figures 3-5 can be regarded as upper bounds on the real errors of the composite line ratios. These median errors can be compared to the theoretical displacement that $R_{\alpha \beta}=3.1$ (instead of 2.86) would imprint on the data. The circle-and-bar traces the intensity and direction (from the circle

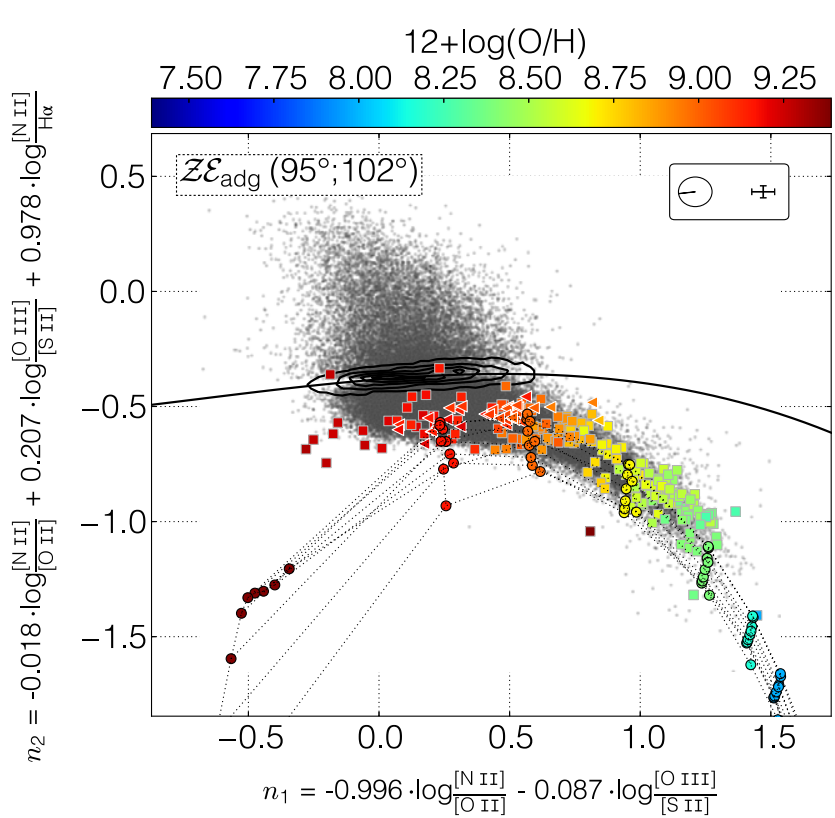

Figure 3. $\mathcal{Z} \mathcal{E}_{\text {adg }}\left(95^{\circ} ; 102^{\circ}\right)$ diagram. The diagram name and associated values of $\left(\phi^{*}, \theta^{*}\right)$ are shown in the top left corner for completeness. H II-like and AGNlike SDSS galaxies are in gray. Uncertain galaxies (based on all $\mathcal{Z} \mathcal{E}$ diagnostics) are represented by density contours $(5 \%, 20 \%, 40 \%$, and $80 \%$ of the maximum density). The round colored dots (connected by the dotted lines) correspond to the MAPPINGS IV models from Dopita et al. (2013). These provide guidance about the theoretical shape of the H II region space. The van Zee et al. (1998) points are represented by small squares with $75 \%$ opacity and the measurements from NGC 5427 are marked with small triangles. All measured H II regions are color-coded according to their oxygen abundance. The black thick line traces our diagnostic line separating the H II-like objects from the AGN-like ones, for which we adopt a third degree polynomial functional form. The black cross (top right) indicates the median error associated with the given combination of SDSS flux ratios, and the circle-and-bar symbol marks the intensity and direction (taken from the circle center outward) of the displacement associated with $R_{\alpha \beta}=3.1$ instead of 2.86 .

(A color version of this figure is available in the online journal.)

center outward) of the total $\zeta$ spatial shift (see Equation (5)). The $\zeta$ shift is always similar to or smaller than the median measurement errors and largely influenced by the $\log [\mathrm{N}$ II $]$ / [O II] ratio.

The values of $\phi^{*}$ and $\theta^{*}$ have been found by interactive inspection of the $\mathcal{Z} \mathcal{Q} \mathcal{E}$ diagrams. ${ }^{10}$ It should be noted here that in all cases, $\phi^{*}$ and $\theta^{*}$ are not tightly constrained. Typically, a variation of $\pm 2^{\circ}$ will not significantly modify the general appearance of the projection, so we restricted our choice to integer values of $\phi^{*}$ and $\theta^{*}$. We find that the theoretical grids created with MAPPINGS IV have a slightly different curvature depending on the chosen value of $\kappa$; for most $\mathcal{Z} \mathcal{E}$ diagrams shown in Figures 3-5, a different value of $\kappa$ could influence the choice of the angles $\phi^{*}$ and $\theta^{*}$ by $\pm 2^{\circ}$. The values quoted in Table 4 are our favored ones for $\kappa=20$.

Our choices of $\phi^{*}$ and $\theta^{*}$ were guided jointly by the appearance of the theoretical models, individual H II regions, and the SDSS starburst branch. Specifically, we first used the model grids to identify a "first-order" point of view from which the grids collapse onto themselves. We then turned our attention to the cloud of SDSS galaxies, and specifically, to the starburst branch, to fine-tune the final choice of $\phi^{*}$ and $\theta^{*}$ so that the observational data appears at its thinnest. For all the $\mathcal{Z} \mathcal{E}$ diagrams but two, the MAPPINGS IV simulation grid (marked 10 The capability to handle 3D models and structures interactively is an
intrinsic characteristic of Mayavi2. 

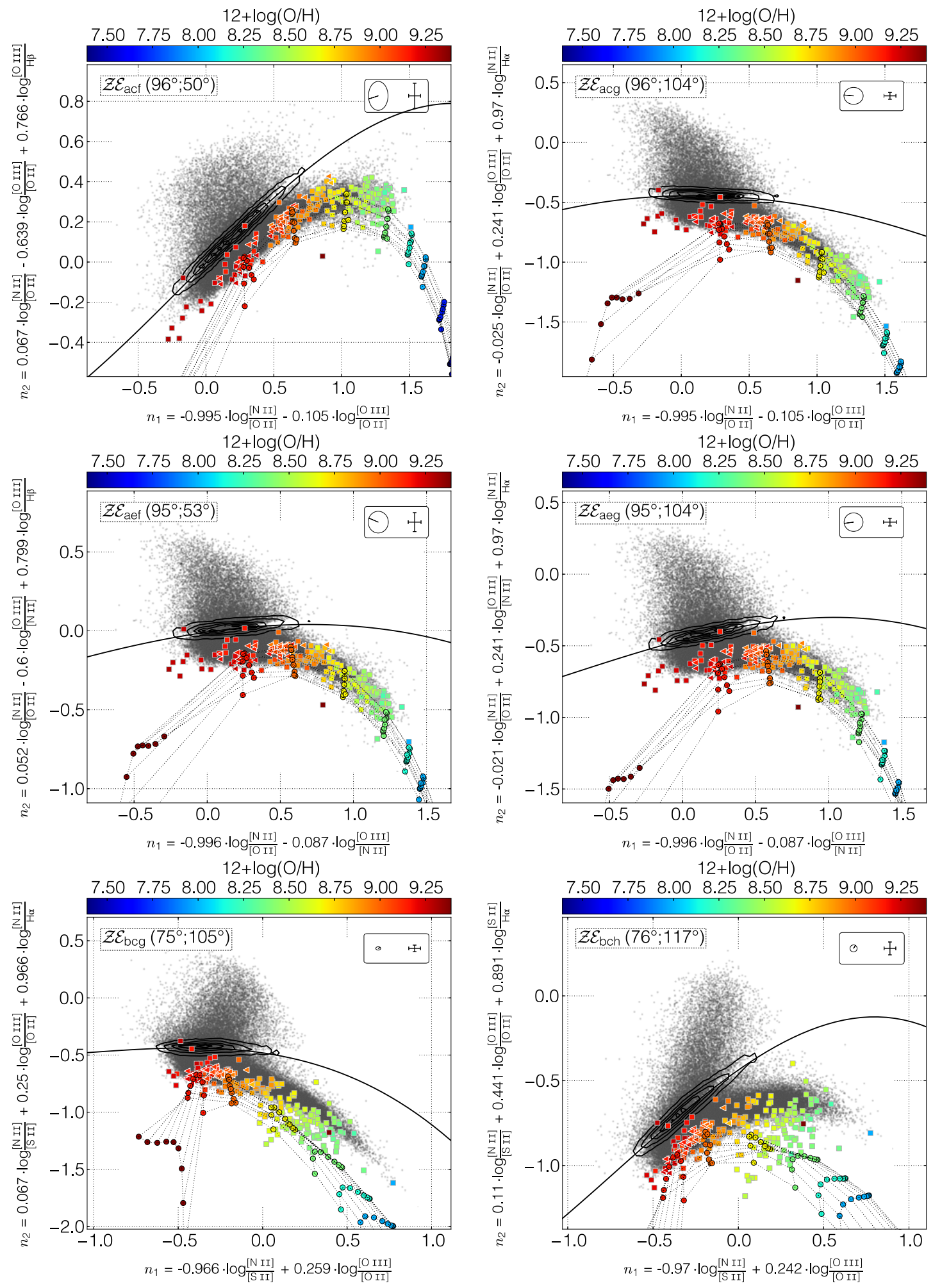

Figure 4. Same as Figure 3, for the other $\mathcal{Z} \mathcal{E}$ diagnostics involving [O II].

(A color version of this figure is available in the online journal.)

by filled circles colored as a function of the corresponding oxygen abundance of the model) is narrow and degenerate, mostly in the $q$ direction. Hence, the $x$ axes of these $\mathcal{Z} \mathcal{E}$ diagrams can be associated with a metallicity $(\mathcal{Z})$ direction. By contrast, most of the differentiation between starburst-like and AGNlike objects is achieved in the $y$ direction, which can therefore be seen as the excitation or $\mathcal{E}$ direction, which is the basis of our chosen nomenclature. For the particular case of the $\mathcal{Z} \mathcal{E}_{\text {beh }}$ and $\mathcal{Z} \mathcal{E}_{\text {bch }}$ diagrams, a $2 \mathrm{D}$ twist inherent to the simulation grid makes it impossible to find a point of view from which the grid collapses for the entire metallicity range. In that case, we chose $\phi^{*}$ and $\theta^{*}$ so that the H II space is most degenerate in the area of largest confusion between HII-like and AGN-like objects. 

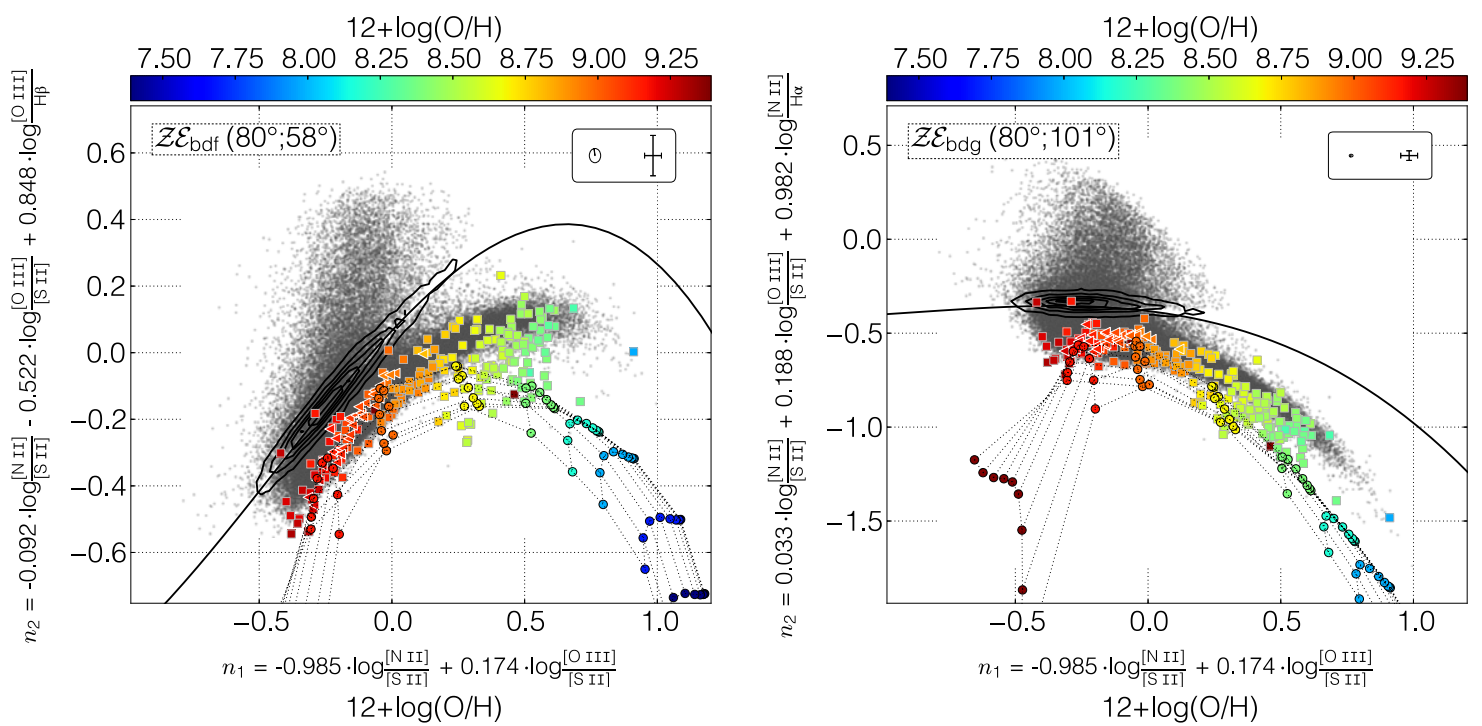

$\begin{array}{llllllll}7.50 & 7.75 & 8.00 & 8.25 & 8.50 & 8.75 & 9.00 & 9.25\end{array}$
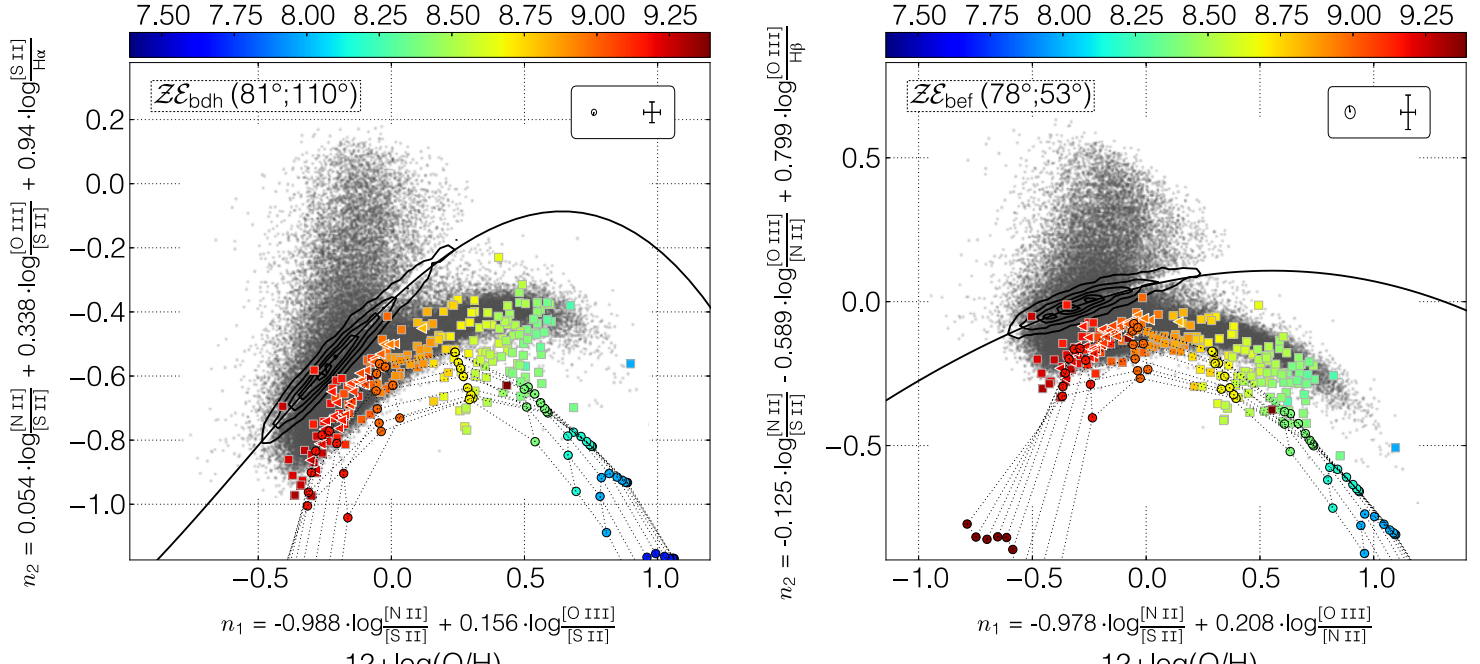

$12+\log (\mathrm{O} / \mathrm{H})$
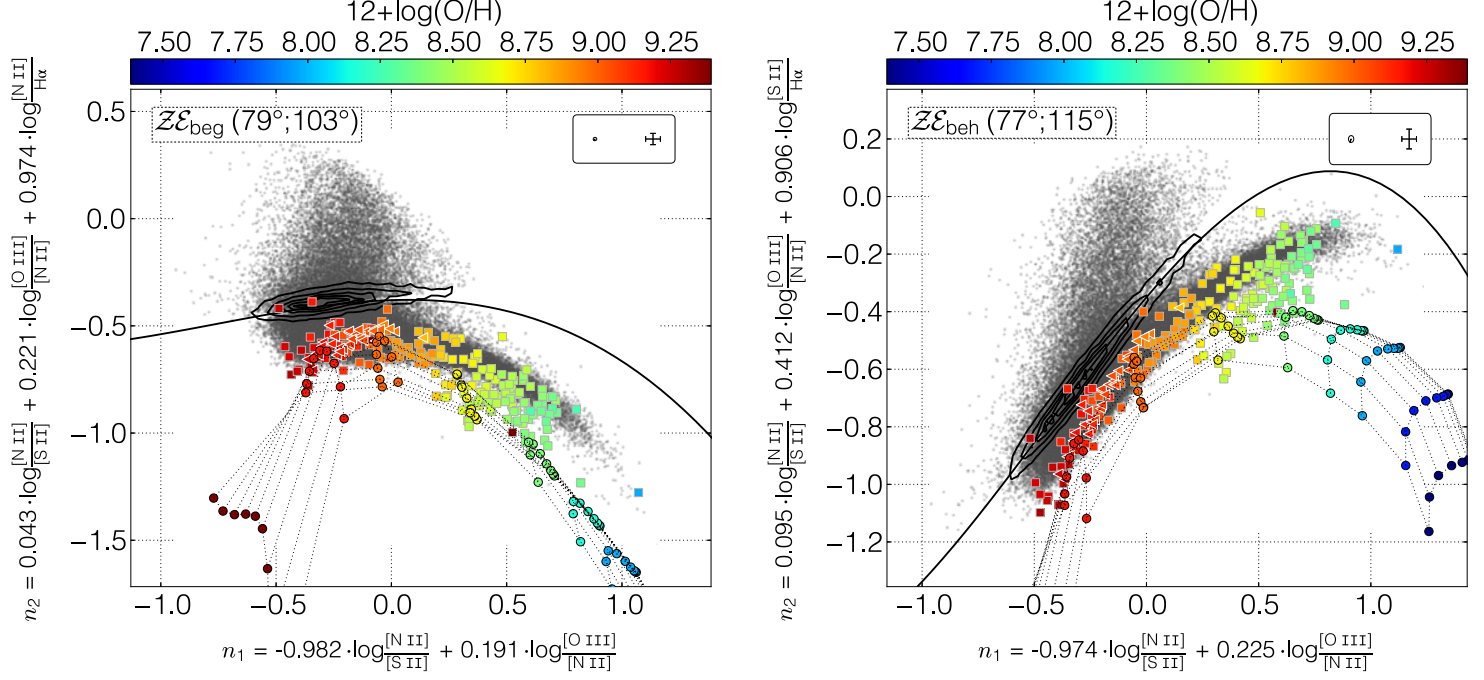

Figure 5. Same as Figure 3, but for the $\mathcal{Z} \mathcal{E}$ diagnostics not involving [O II]

(A color version of this figure is available in the online journal.)

Identifying a specific viewpoint on the 3D distribution of SDSS observational data points is somewhat reminiscent of the notion of the fundamental plane (FP) for early-type galaxies (Dressler et al. 1987; Djorgovski \& Davis 1987). In that situation, the identification of the parameters of the best-fit FP is often performed automatically, for example, by computing the direction of smallest scatter in the data (e.g., Jorgensen et al. 1996), or with similar but more sophisticated approaches (e.g., Bernardi et al. 2003; Saulder et al. 2013). While in principle it is not impossible to perform an analytical identification of $\phi^{*}$ and $\theta^{*}$, in practice it is less straightforward than our adopted manual solution. First, the structure of the $3 \mathrm{D}$ distribution of 
Table 4

$\mathcal{Z E}$ Diagrams and their Associated Parameters

\begin{tabular}{|c|c|c|c|c|}
\hline Name & $\phi^{*}$ & $\theta^{*}$ & $n_{1}$ & $n_{2}$ \\
\hline $\mathcal{Z} \mathcal{E}_{\text {acf }}$ & 96 & 50 & $-0.995 \times \log \frac{[\mathrm{N} \mathrm{II}]}{[\mathrm{O} \mathrm{III}]}-0.105 \times \log \frac{[\mathrm{O} \mathrm{III}]}{[\mathrm{O} \mathrm{II}]}$ & $+0.067 \times \log \frac{[\mathrm{N} \mathrm{II}]}{[\mathrm{O} \mathrm{II}]}-0.639 \times \log \frac{[\mathrm{O} \mathrm{III}]}{[\mathrm{O} \mathrm{II}]}+0.766 \times \log \frac{[\mathrm{O} \mathrm{III}]}{[\mathrm{H} \beta]}$ \\
\hline $\mathcal{Z} \mathcal{E}_{\text {acg }}$ & 96 & 104 & $-0.995 \times \log \frac{[\mathrm{N} \mathrm{III}]}{[\mathrm{O} \mathrm{III}]}-0.105 \times \log \frac{[\mathrm{O} \mathrm{IIII}]}{[\mathrm{O} \mathrm{II}]}$ & $-0.025 \times \log \frac{[\mathrm{N} \mathrm{II}]}{[\mathrm{O} \mathrm{II}]}+0.241 \times \log \frac{[\mathrm{O} \mathrm{III}]}{[\mathrm{O} \mathrm{III}]}+0.970 \times \log \frac{[\mathrm{N} \mathrm{II}]}{[\mathrm{H} \alpha]}$ \\
\hline $\mathcal{Z} \mathcal{E}_{\text {adg }}$ & 95 & 102 & $-0.996 \times \log \frac{[\mathrm{N} \mathrm{II}]}{[\mathrm{OII}]}-0.087 \times \log \frac{[\mathrm{O} \mathrm{IIII}]}{[\mathrm{OII}]}$ & $-0.018 \times \log \frac{[\mathrm{N} \mathrm{III}]}{[\mathrm{O} \mathrm{II}]}+0.207 \times \log \frac{[\mathrm{O} \mathrm{III}]}{[\mathrm{OII}]}+0.978 \times \log \frac{[\mathrm{N} \mathrm{II}]}{[\mathrm{H} \alpha]}$ \\
\hline $\mathcal{Z} \mathcal{E}_{\text {aef }}$ & 95 & 53 & $-0.996 \times \log \frac{[\mathrm{N} \mathrm{II}]}{[\mathrm{OII}]}-0.087 \times \log \frac{[\mathrm{O} \mathrm{III}]}{[\mathrm{NII}]}$ & $+0.052 \times \log \frac{[\mathrm{N} \mathrm{III}]}{[\mathrm{OII}]}-0.600 \times \log \frac{[\mathrm{OIII}]}{[\mathrm{N} \mathrm{II}]}+0.799 \times \log \frac{[\mathrm{O} \mathrm{III}]}{[\mathrm{H} \beta]}$ \\
\hline $\mathcal{Z} \mathcal{E}_{\text {aeg }}$ & 95 & 104 & $-0.996 \times \log \frac{[\mathrm{N} \mathrm{II}]}{[\mathrm{OII}]}-0.087 \times \log \frac{[\mathrm{OIII}]}{[\mathrm{N} \mathrm{II}]}$ & $-0.021 \times \log \frac{[\mathrm{N} \mathrm{III}]}{[\mathrm{OII}]}+0.241 \times \log \frac{[\mathrm{O} \mathrm{III}]}{[\mathrm{N} \mathrm{II}]}+0.970 \times \log \frac{[\mathrm{N} \mathrm{II}]}{[\mathrm{H} \alpha]}$ \\
\hline $\mathcal{Z} \mathcal{E}_{\text {bcg }}$ & 75 & 105 & $-0.966 \times \log \frac{[\mathrm{N} \mathrm{II}]}{[\mathrm{S} \mathrm{II}]}+0.259 \times \log \frac{[\mathrm{OIII}]}{[\mathrm{OII}]}$ & $+0.067 \times \log \frac{[\mathrm{N} \mathrm{II}]}{[\mathrm{S} \mathrm{II}]}+0.250 \times \log \frac{[\mathrm{OIII}]}{[\mathrm{OII}]}+0.966 \times \log \frac{[\mathrm{N} \mathrm{II}]}{[\mathrm{H} \alpha]}$ \\
\hline $\mathcal{Z} \mathcal{E}_{\text {bch }}$ & 76 & 117 & $-0.970 \times \log \frac{[\mathrm{N} \mathrm{II}]}{[\mathrm{S} \mathrm{III}]}+0.242 \times \log \frac{[\mathrm{O} \mathrm{III}]}{[\mathrm{OII}]}$ & $+0.110 \times \log \frac{[\mathrm{N} \mathrm{II}]}{[\mathrm{S} \mathrm{II}]}+0.441 \times \log \frac{[\mathrm{O} \mathrm{III}]}{[\mathrm{OII}]}+0.891 \times \log \frac{[\mathrm{N} \mathrm{II}]}{[\mathrm{H} \alpha]}$ \\
\hline $\mathcal{Z} \mathcal{E}_{\text {bdf }}$ & 80 & 58 & $-0.985 \times \log \frac{[\mathrm{NII}]}{[\mathrm{SII}]}+0.174 \times \log \frac{[\mathrm{OIII}]}{[\mathrm{SII}]}$ & $-0.092 \times \log \frac{[\mathrm{N} \mathrm{III}]}{[\mathrm{S} \mathrm{II}]}-0.522 \times \log \frac{[\mathrm{O} \mathrm{III}]}{[\mathrm{S} \mathrm{II}]}+0.848 \times \log \frac{[\mathrm{O} \mathrm{III}]}{[\mathrm{H} \beta]}$ \\
\hline $\mathcal{Z} \mathcal{E}_{\text {bdg }}$ & 80 & 101 & $-0.985 \times \log \frac{[\mathrm{NII}]}{[\mathrm{SII}]}+0.174 \times \log \frac{[\mathrm{OIII}]}{[\mathrm{SII}]}$ & $+0.033 \times \log \frac{[\mathrm{NII}]}{[\mathrm{SII}]}+0.188 \times \log \frac{[\mathrm{O} \mathrm{III}]}{[\mathrm{S} \mathrm{II}]}+0.982 \times \log \frac{[\mathrm{N} \mathrm{II}]}{[\mathrm{H} \alpha]}$ \\
\hline $\mathcal{Z} \mathcal{E}_{\text {bdh }}$ & 81 & 110 & $-0.988 \times \log \frac{[\mathrm{NII}]}{[\mathrm{SII}]}+0.156 \times \log \frac{[\mathrm{OIII}]}{[\mathrm{S} \mathrm{II}]}$ & $+0.054 \times \log \frac{[\mathrm{N} \mathrm{II}]}{[\mathrm{SII}]}+0.338 \times \log \frac{[\mathrm{O} \mathrm{III}]}{[\mathrm{S} \mathrm{II}]}+0.940 \times \log \frac{[\mathrm{S} \mathrm{II}]}{[\mathrm{H} \alpha]}$ \\
\hline $\mathcal{Z} \mathcal{E}_{\text {bef }}$ & 78 & 53 & $-0.978 \times \log \frac{[\mathrm{NII}]}{[\mathrm{SII}]}+0.208 \times \log \frac{[\mathrm{OIII}]}{[\mathrm{N} \mathrm{II}]}$ & $-0.125 \times \log \frac{[\mathrm{N} \mathrm{III}]}{[\mathrm{S} \mathrm{II}]}-0.589 \times \log \frac{[\mathrm{O} \mathrm{III}]}{[\mathrm{NII}]}+0.799 \times \log \frac{[\mathrm{O} \mathrm{III}]}{[\mathrm{H} \beta]}$ \\
\hline $\mathcal{Z} \mathcal{E}_{\text {beg }}$ & 79 & 103 & $-0.982 \times \log \frac{[\mathrm{N} \mathrm{III}]}{[\mathrm{S} \mathrm{II}]}+0.191 \times \log \frac{[\mathrm{O} \mathrm{III}]}{[\mathrm{N} \mathrm{II}]}$ & $+0.043 \times \log \frac{[\mathrm{N} \mathrm{II}]}{[\mathrm{S} \mathrm{II}]}+0.221 \times \log \frac{[\mathrm{O} \mathrm{III}]}{[\mathrm{NII}]}+0.974 \times \log \frac{[\mathrm{N} \mathrm{II}]}{[\mathrm{H} \alpha]}$ \\
\hline $\mathcal{Z} \mathcal{E}_{\text {beh }}$ & 77 & 115 & $-0.974 \times \log \frac{[\mathrm{N} \mathrm{III}]}{[\mathrm{S} \mathrm{II}]}+0.225 \times \log \frac{[\mathrm{O} I \mathrm{III}]}{[\mathrm{NII}]}$ & $+0.095 \times \log \frac{[\mathrm{N} \mathrm{II}]}{[\mathrm{S} \mathrm{II}]}+0.412 \times \log \frac{[\mathrm{O} \mathrm{III}]}{[\mathrm{NII}]}+0.906 \times \log \frac{[\mathrm{S} \mathrm{II}]}{[\mathrm{H} \alpha]}$ \\
\hline
\end{tabular}

SDSS galaxies in the $\mathcal{Z} \mathcal{Q E}$ diagrams is significantly more complex than that of a plane. Second, the data set contains both H II-like and AGN-like objects, but in the present case one is only interested in collapsing the starburst branch onto itself, not the entire cloud of data points. If it is possible to identify and track the location of the starburst branch "by eye," it is significantly more complex to do so analytically and without any prior knowledge of the classification of the different objects.

As we have found manually, any choice of $\phi^{*}$ and $\theta^{*}$ is not tightly constrained, and could vary by $\pm 2^{\circ}$ without significantly affecting the structure of the $\mathcal{Z E}$ diagram. Under this circumstance and at this point in time, our manual identification of $\phi^{*}$ and $\theta^{*}$ appears as satisfactory and useful as any analytical approach. Particularly, analytical determinations of $\phi^{*}$ and $\theta^{*}$ would still depend on the underlying data set and the chosen methodology, and would therefore not be "unique" (as is the case for the FP parameters; see Bernardi et al. 2003). The implementation of an automated routine to identify $\phi^{*}$ and $\theta^{*}$ is outside the scope of this paper, but ought to be explored in the future as the quality of the observational data points and theoretical data sets improves further. For example, a spaxel-based analysis relying on ongoing or upcoming IFU surveys such as Califa (Sánchez et al. 2012), SAMI (Croom et al. 2012), or MANGA could better differentiate between the core and the outskirts of galaxies and possibly reduce the inherent confusion at the interface between star-formation-dominated and AGNdominated objects (Maragkoudakis et al. 2014; Davies et al. 2014).

Principal component analysis (PCA) is a statistical technique that can identify directions of interest in multi-dimensional data sets by calculating the successive normal directions of maximum variance (see, e.g., Francis \& Wills 1999, for a brief introduction). When performing a PCA analysis, the main challenge resides in interpreting these directions of interest and connecting them to the physical world. The approach we adopt for creating the $\mathcal{Z} \mathcal{E}$ diagrams (and associated diagnostics) follows the opposite path. Here, we use direct physical insight to separate line ratios into three complementary categories, and only then, once we have constructed the corresponding $\mathcal{Z Q E}$ space, inspect it interactively to find points of view of interest. The interactive aspect of our approach is especially useful in allowing us to simultaneously compare the grids of theoretical
Table 5

Starburst Diagnostic Line Parameters for Each $\mathcal{Z E}$ Diagram

\begin{tabular}{lcccc}
\hline \hline Name & $\alpha$ & $\beta$ & $\gamma$ & $\delta$ \\
\hline $\mathcal{Z} \mathcal{E}_{\text {acf }}$ & -0.059 & -0.024 & +0.676 & -0.005 \\
$\mathcal{Z} \mathcal{E}_{\text {acg }}$ & +0.005 & -0.124 & +0.020 & -0.445 \\
$\mathcal{Z} \mathcal{E}_{\text {adg }}$ & -0.034 & -0.071 & +0.091 & -0.382 \\
$\mathcal{Z} \mathcal{E}_{\text {aef }}$ & -0.013 & -0.082 & +0.133 & -0.008 \\
$\mathcal{Z} \mathcal{E}_{\text {aeg }}$ & -0.032 & -0.079 & +0.268 & -0.459 \\
$\mathcal{Z} \mathcal{E}_{\text {bcg }}$ & -0.101 & -0.311 & -0.216 & -0.481 \\
$\mathcal{Z} \mathcal{E}_{\text {bch }}$ & -0.132 & -0.280 & +0.700 & -0.437 \\
$\mathcal{Z} \mathcal{E}_{\text {bdf }}$ & -0.283 & -0.368 & +0.851 & +0.066 \\
$\mathcal{Z} \mathcal{E}_{\text {bdg }}$ & -0.118 & -0.307 & -0.171 & -0.382 \\
$\mathcal{Z} \mathcal{E}_{\text {bdh }}$ & -0.238 & -0.374 & +0.774 & -0.366 \\
$\mathcal{Z} \mathcal{E}_{\text {bef }}$ & -0.013 & -0.157 & +0.186 & +0.055 \\
$\mathcal{Z} \mathcal{E}_{\text {beg }}$ & -0.097 & -0.222 & +0.034 & -0.380 \\
$\mathcal{Z} \mathcal{E}_{\text {beh }}$ & -0.221 & -0.289 & +0.920 & -0.350 \\
\hline
\end{tabular}

models, the individual measurements of $\mathrm{H}$ II regions, and SDSS galaxies. Of course, the prime advantage of PCA is that it is not restricted to 3D spaces. That is, a PCA analysis could be applied to the entire multi-dimensional line ratio space of galaxies, unlike the $\mathcal{Z} \mathcal{Q E}$ diagram approach, which, for obvious reasons, cannot probe beyond 3D. Hence, while a detailed comparison is outside the scope of this article, the PCA approach and the $\mathcal{Z} \mathcal{Q E}$ approach are (conceptually) very complimentary.

In any situation, to avoid misunderstandings and ensure repeatability, we strongly advise any use of the $\mathcal{Z E}$ diagrams to clearly state the values of $\phi$ and $\theta$ employed along with the line ratios involved, which are required to uniquely define any $\mathcal{Z E}$ diagram (see Section 5.1 and Equation (6)).

\subsubsection{Defining the Diagnostic Lines}

For each $\mathcal{Z E}_{\mathrm{x}_{1} \mathrm{x}_{2} \mathrm{x}_{3}}\left(\phi^{*} ; \theta^{*}\right)$ diagram illustrated in Figures 3-5, we define a third-order polynomial that separates the Hir-like objects (below the line) and the AGN-like objects (above the line). The semi-empirical polynomial coefficients $\alpha, \beta, \gamma$ and $\delta$ are summarized in Table 5, where the line equation is defined by

$$
y=f\left(n_{1}\right)=\alpha\left(n_{1}\right)^{3}+\beta\left(n_{1}\right)^{2}+\gamma\left(n_{1}\right)+\delta .
$$

This approach is similar to that used by Kewley et al. (2001a) and Kauffmann et al. (2003a) to define diagnostic lines for the 
classical optical line ratio diagnostic diagrams, although the chosen functional forms are different.

The theoretical grids do not match the envelope of the observations of $\mathrm{H}$ II regions perfectly (see Figures 3-5). This is especially true for $\mathcal{Z} \mathcal{E}$ diagrams involving the [S II] lines, which, as noted by Dopita et al. (2013), appear to be $0.1 \mathrm{dex}$ too weak in the models. Several possible origins exist for the theoretical mismatch. At the low abundance end in particular, some $\mathrm{H}$ II regions may possibly have a higher electron density than expected (up to $n_{e} \simeq 100 \mathrm{~cm}^{3}$; Nicholls et al. 2014). At the high-abundance end, all lines become very sensitive to the electron temperature, which varies very rapidly through the models. Thus, small changes in the geometry of the ionized gas (assumed to be spherically symmetric in the models) can make large differences in the predicted emission line spectrum. The underlying stellar synthesis models may also be largely responsible for the offset between the theoretical grids and the SDSS galaxies (especially for line ratios involving the [S II] lines) if these theoretical models do not produce enough far-UV ionizing photons, as suggested by Kewley et al. (2001a) and Levesque et al. (2010). Lastly, we note that the spacing between the two highest abundance sets of simulations are $\sim 2-3$ times larger than the spacing between the other abundance sets. As a result, linearly interpolating (as traced by the dotted lines in Figures 3-5) can be a poorer estimation and result in a larger mismatch between the theoretical grid and the observations.

Given the mismatch between the shape of the model grids and the observational data points in some of the $\mathcal{Z} \mathcal{E}$ diagrams, we use the theoretical models as a general guide, but choose the final coefficients $\alpha, \beta, \gamma$, and $\delta$ so that the diagnostic lines trace the full extent of the starburst sequence of the SDSS galaxies in all cases. Hence, keeping in mind that we indirectly rely on the theoretical models in the manual determination procedure for the values of $\phi^{*}$ and $\theta^{*}$ (see Section 5.2.1), the different $\mathcal{Z}$ diagnostics do not depend explicitly on the MAPPINGS IV grids.

In practice, the diagnostic line coefficients are identified as follows. We first manually choose a series of five to seven positions in the $\mathcal{Z} \mathcal{E}$ diagram, spaced by $0.2-0.5$ dex along the $x$ direction, defining a first-order separation between $\mathrm{H}$ II-like and AGN-like objects. We subsequently obtain the corresponding polynomial coefficients by performing a least-square minimization of a third order polynomial for these data points using the Python implementation of the IDL ${ }^{11}$ nonlinear least-square minimization routine mpfit (Markwardt 2009). Since we set these diagnostic lines manually and independently for each diagram, using the theoretical grid for guidance only, and given that each diagnostic is subject to both observational errors and theoretical uncertainties, it is possible that an SDSS galaxy classified as $\mathrm{H}$ II-like by one diagnostic will be classified as AGN-like by others. However, because we now have 13 diagnostics, we can combine them to ensure consistency and reduce the classification uncertainty.

To that end, we separate all SDSS galaxies into three groups:

1. HII-like. Galaxies classified as $\mathrm{H}$ II-like by all $13 \mathcal{Z E}$ diagnostics.

2. AGN-like. Galaxies classified as AGN-like by all $13 \mathcal{Z E}$ diagnostics.

3. Uncertain. Galaxies for which the $13 \mathcal{Z E}$ diagnostics are inconsistent.

11 Interactive Data Language.
In Figures 3-5, density contours delineate the location of SDSS galaxies having an uncertain classification. The contours have been obtained by distributing all the galaxies with uncertain classification in a regular grid with a resolution of $0.03 \mathrm{dex}$, with subsequent smoothing of the grid with a symmetric Gaussian filter of 0.15 dex in radius (five grid elements). As can be expected, the uncertain galaxies are clustered around each of the diagnostic lines, with the $20 \%$ contour within \pm 0.1 dex of the diagnostic line. Using a manual and iterative approach, we have adapted the parameters of each of the diagnostic equations to minimize the number of uncertain galaxies. Following this approach, we reduced the number of galaxies with uncertain classification to 2636 ( $2.5 \%$ of a total of 105,070 objects). We have $88,918 \mathrm{H}$ II-like galaxies $(84.6 \%)$ and 13,516 AGN-like galaxies $(12.9 \%)$, as classified by the $\mathcal{Z} \mathcal{E}$ diagnostics.

Improving the overall agreement between the different diagnostics required in some cases to alter the shape of the diagnostic lines, especially for high metallicities. Because the SDSS points are not distributed uniformly, very small modifications of the diagnostic line in denser regions can strongly influence the overall agreement of the different diagnostics. Since we rely on third order polynomials, the innermost regions of the diagnostic lines are very much influenced by the slope at higher (and lower) metallicities. In other words, the lack of observations makes it impossible to tightly constrain the position of the diagnostic line in the outermost region of the different $\mathcal{Z} \mathcal{Q} \mathcal{E}$ diagrams.

We examine the consistency of each of these $\mathcal{Z} \mathcal{E}$ diagnostics in more detail in the next subsection. This is not to be confused with the validity of the final classification itself, which we examine in Section 6.1. In Appendix A, we show for completeness the $\mathcal{Z} \mathcal{E}$ diagrams that best collapse the grid of photoionization models in the $11 \mathcal{Z} \mathcal{Q E}$ spaces for which we did not derive any $\mathcal{Z} \mathcal{E}$ diagnostic. These diagrams were not selected as reliable diagnostics because of the high confusion between the starburst and AGN branches of the SDSS galaxies. In Figures 13 and 14, the confusion is emphasized by showing the density contours of galaxies with uncertain classification. Although the final $\mathcal{Z} \mathcal{E}$ classification and hence the density contours of uncertain galaxies were derived "after" the visual selection of $\mathcal{Z} \mathcal{Q E}$ diagrams with a clean separation between the starburst and AGN branches of SDSS galaxies, these contours act as an a posteriori confirmation of the initial selection. In every $\mathcal{Z} \mathcal{E}$ diagram shown in Figures 13 and 14 the uncertain galaxies spread out over large areas $(>0.2$ dex $)$, unlike in the $\mathcal{Z E}$ diagnostic diagrams listed in Table 4.

\subsection{Consistency of the $\mathcal{Z} \mathcal{E}$ Diagnostics}

The $13 \mathcal{Z E}$ diagnostics defined in Table 5 all rely on a subset of nine line ratios, so that they are not strictly independent from one another. To better understand this connection, we focus our attention on the $2636(2.5 \%)$ galaxies with an uncertain classification. We introduce the quantity $\eta\left(\mathcal{Z} \mathcal{E}_{\mathrm{x}_{1} \mathrm{x}_{2} \mathrm{x}_{3}}\right)$ as the percentage of uncertain galaxies classified as AGN-like by a particular $\mathcal{Z E}_{\mathrm{x}_{1} \mathrm{x}_{2} \mathrm{x}_{3}}$ diagnostic. The value of $\eta$ for the 13 $\mathcal{Z} \mathcal{E}$ diagnostics is shown in Figure 6. A low value of $\eta$ indicates a diagnostic that is too lax and will classify most uncertain galaxies as $\mathrm{H}$ II-like. On the other hand, a high value of $\eta$ indicates a diagnostic that is too tight. In such a case, the majority of the uncertain galaxies are classified as being AGN-like by the diagnostic concerned. All $13 \mathcal{Z E}$ diagnostics have $40 \%<\eta\left(\mathcal{Z E}_{\mathrm{x}_{1} \mathrm{x}_{2} \mathrm{x}_{3}}\right)<60 \%$, an indication that overall, they are all consistent with each other (as expected from our iterative procedure to define the $\alpha, \beta, \gamma$, and $\delta$ coefficients; see 


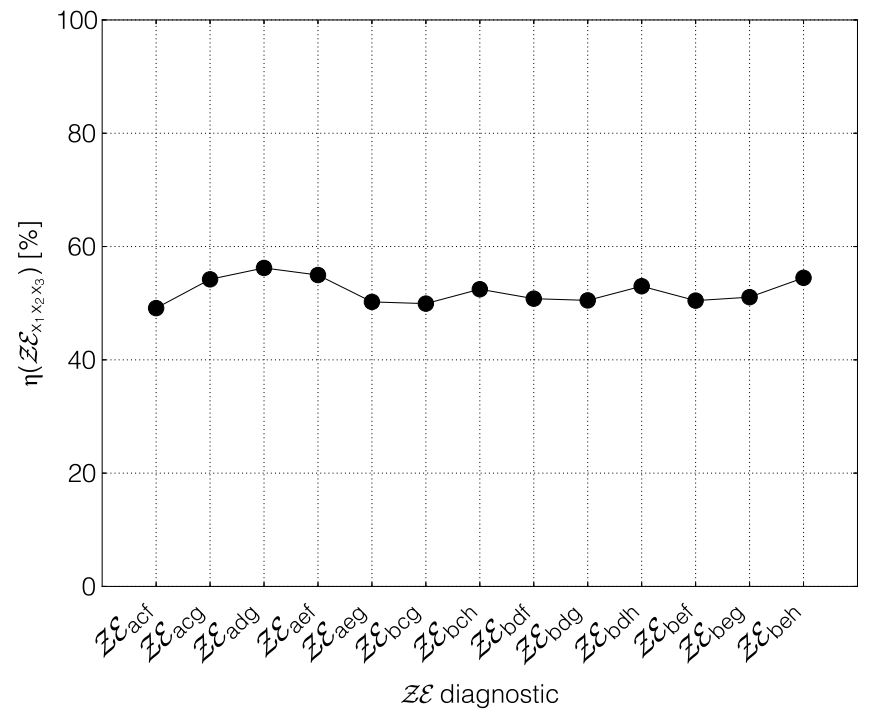

Figure 6. $\eta\left(\mathcal{Z} \mathcal{E}_{\mathrm{x}_{1} \mathrm{x}_{2} \mathrm{x}_{3}}\right)$, the percentage of uncertain galaxies classified as AGNlike by a particular $\mathcal{Z} \mathcal{E}_{\mathrm{x}_{1} \mathrm{x}_{2} \mathrm{x}_{3}}$ diagnostic with respect to the total number of galaxies with uncertain classification.

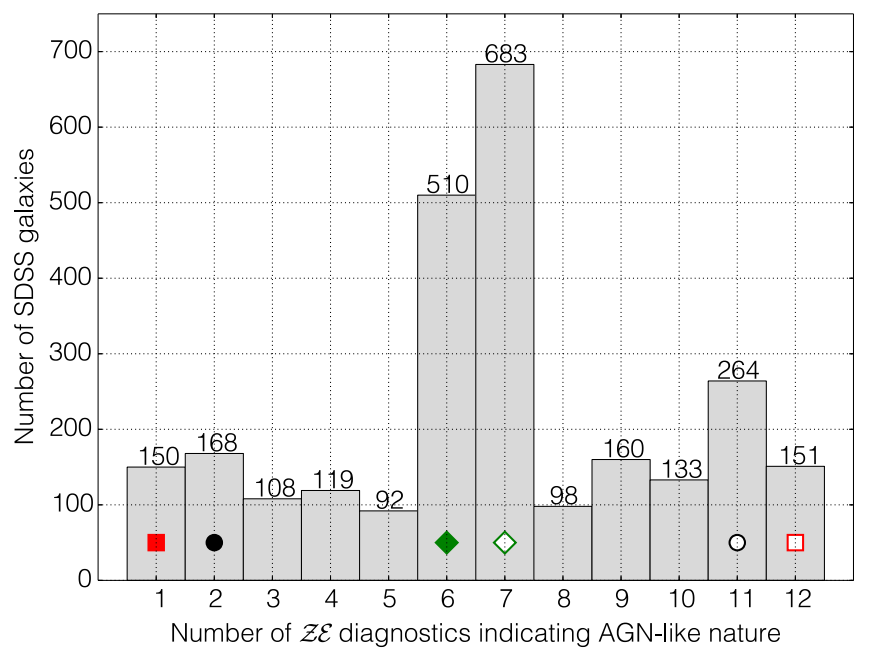

Figure 7. Distribution of the number of uncertain galaxies as a function of the number of $\mathcal{Z E}$ diagnostics indicating that they are AGN-like. The symbols in bins "1", "2", "6", "7", "11,"and "12" correspond to the different lines in Figure 8 and are shown here for completeness.

(A color version of this figure is available in the online journal.)

Section 5.2.2). The underlying distribution of SDSS galaxies is not uniform, and small "local" displacements of the diagnostic lines in the $\mathcal{Z} \mathcal{E}$ diagram can easily give rise to the range of $\eta$ values shown in Figure 6.

A complimentary way to look at the SDSS galaxies with uncertain classification is illustrated in Figure 7, where we show the distribution of the number of uncertain galaxies as a function of how many $\mathcal{Z E}$ diagnostics indicate that they are AGN-like. For example, the bin "1" corresponds to galaxies

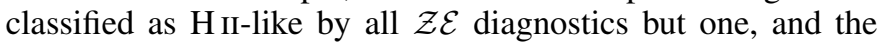
bin "12" corresponds to galaxies classified as AGN-like by all $\mathcal{Z E}$ diagnostics but one. The distribution harbors a sharp peak at $\sim 6.5$ and a secondary, minor peak at 11 , in addition to a base level of $\sim 130$ objects per bin. The base level of uncertain galaxies in all bins is most certainly a consequence of the small mismatch between the different diagnostics as well as observational errors of certain line ratios.

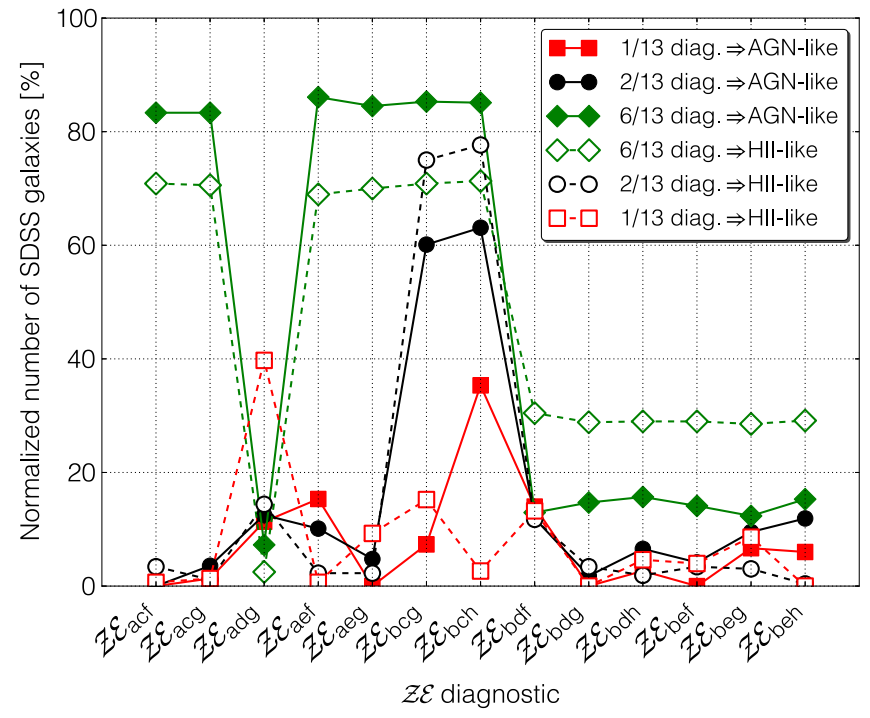

Figure 8. For each $\mathcal{Z} \mathcal{E}_{\mathrm{x}_{1} \mathrm{x}_{2} \mathrm{x}_{3}}$ diagnostic, the normalized number of uncertain galaxies for which this $\mathcal{Z} \mathcal{E}$ diagnostic is discordant, when all diagnostics but one agree (squares), all diagnostics but two agree (circles), and only six diagnostics agree (diamonds). Full symbols correspond to uncertain objects classified as $\mathrm{H}$ II-like by most $\mathcal{Z} \mathcal{E}$ diagnostics, and empty symbols correspond to objects classified as AGN-like by most $\mathcal{Z} \mathcal{E}$ diagnostics.

(A color version of this figure is available in the online journal.)

The existence of the central and secondary peaks in the histogram shown in Figure 7 are perhaps more interesting. To understand their origin, we compute in Figure 8 (for each $\mathcal{Z E}$ diagnostic) the normalized number of uncertain galaxies for which this $\mathcal{Z E}$ diagnostic is discordant. In other words, we ask whether some diagnostics are more discordant than the others. For clarity, we restrict ourselves to the data corresponding to bins 1, 2, 6, 7, 11, and 12 in Figure 7, that is, objects that have only one (square symbols), two (circles), and six (diamonds) discordant diagnostics.

First, we note that $\mathcal{Z} \mathcal{E}_{\text {bcg }}$ and $\mathcal{Z} \mathcal{E}_{\text {bch }}$ are responsible for $\sim 75 \%$ of galaxies with only two discordant diagnostics, and are thereby mostly responsible for the secondary peak in bin 11 of Figure 7 . This is a first indication of the somewhat poorer quality of these two diagnostics compared to the other ones.

Concerning the uncertain galaxies with six discordant $\mathcal{Z E}$ diagnostics (diamond symbols), we detect a clear dichotomy. On the one hand, $\left[\mathcal{Z} \mathcal{E}_{\text {acf }} ; \mathcal{Z} \mathcal{E}_{\text {acg }} ; \mathcal{Z} \mathcal{E}_{\text {aef }} ; \mathcal{Z} \mathcal{E}_{\text {aeg }} ; \mathcal{Z} \mathcal{E}_{\text {bcg }} ; \mathcal{Z} \mathcal{E}_{\text {bch }}\right]$ are each in disagreement with the dominant classification in $\sim 70 \%-85 \%$ of the cases. On the other hand, $\left[\mathcal{Z} \mathcal{E}_{\text {bdf }} ; \mathcal{Z} \mathcal{E}_{\text {bdg }}\right.$; $\left.\mathcal{Z} \mathcal{E}_{\text {bdh }} ; \mathcal{Z E}_{\text {bef }} ; \mathcal{Z} \mathcal{E}_{\text {beg }} ; \mathcal{Z} \mathcal{E}_{\text {beh }}\right]$ are concordant with the dominant classification in $\sim 70 \%-85 \%$ of the cases. $\mathcal{Z} \mathcal{E}_{\text {adg }}$ is almost always ( $\sim 95 \%)$ consistent with the dominant classification. The overall consistency between the two groups of $\mathcal{Z E}$ diagnostics is suggestive of a possible underlying correlation. To explore this possibility, we show in Figure 9 the agreement matrix between each pair of $\mathcal{Z E}$ diagnostics. A high value indicates that two diagnostics tend to be concordant in their classification of uncertain galaxies $(100 \%=$ always in agreement $)$, while a lower value indicates that the two diagnostics mostly disagree on the classification of uncertain galaxies $(0 \%=$ never in agreement).

Indeed, we detect a clear correlation between the two sets of diagnostics

$$
\left[\mathcal{Z E}_{\text {acf }} ; \mathcal{Z} \mathcal{E}_{\text {acg }} ; \mathcal{Z} \mathcal{E}_{\text {aef }} ; \mathcal{Z} \mathcal{E}_{\text {aeg }} ; \mathcal{Z} \mathcal{E}_{\text {bcg }} ; \mathcal{Z} \mathcal{E}_{\text {bch }}\right]
$$




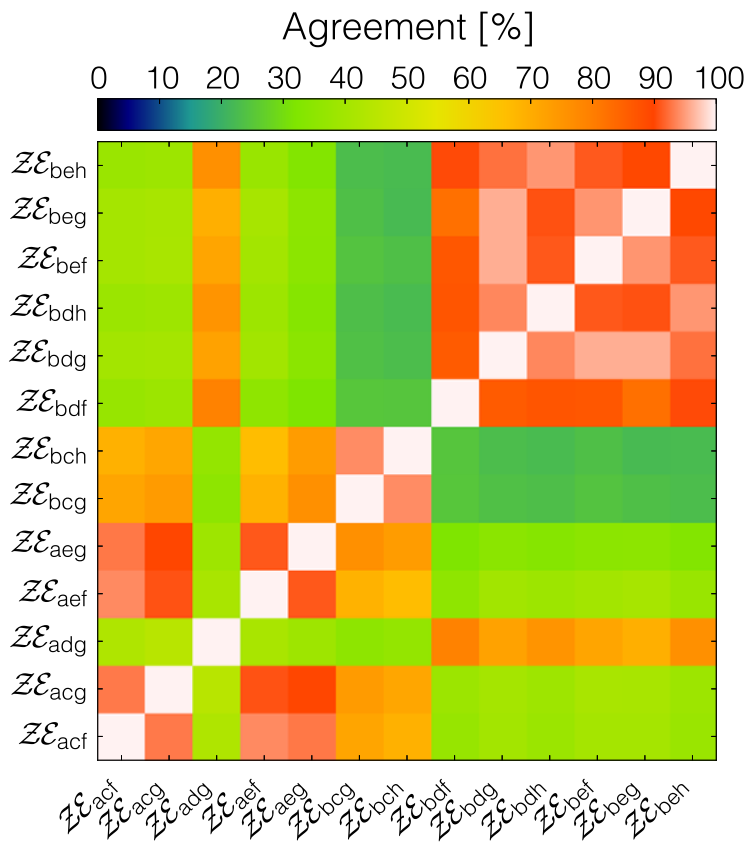

Figure 9. Level of agreement between the different $\mathcal{Z} \mathcal{E}$ diagnostics regarding the classification of uncertain galaxies.

(A color version of this figure is available in the online journal.)

and

$$
\left[\mathcal{Z} \mathcal{E}_{\text {adg }} ; \mathcal{Z E}_{\text {bdf }} ; \mathcal{Z} \mathcal{E}_{\text {bdg }} ; \mathcal{Z} \mathcal{E}_{\text {bdh }} ; \mathcal{Z} \mathcal{E}_{\text {bef }} ; \mathcal{Z} \mathcal{E}_{\text {beg }} ; \mathcal{Z} \mathcal{E}_{\text {beh }}\right]
$$

The level of agreement within each group is high ( $\sim 75 \%-95 \%)$, but the agreement between the two groups overall is much poorer, of the order of $\sim 25 \%-45 \%$. This strong dichotomy is giving rise to the central peak in the distribution of uncertain objects in Figure 7.

This bimodal grouping of the $\mathcal{Z E}$ diagnostics is most certainly no accident, as it separates $\mathcal{Z E}$ diagnostic involving [O II] from the others, with $\mathcal{Z} \mathcal{E}_{\text {adg }}$ the only exception. The fact that diagnostics involving [O II] are more discordant than other ones is strongly suggestive of reddening errors. Most likely, such reddening correction errors in our sample are responsible for this dichotomy, and responsible for most of the $510+683=1193$ galaxies with six discordant diagnostics. As expected and shown in Figures 3-5 via the $\zeta$ shift, most $\mathcal{Z E}$ diagnostics involving [O II] are more sensitive to such reddening corrections. We also note that the overall sensitivity of the SDSS spectrograph is decreasing sharply below $\sim 4000 \AA$ (Smee et al. 2013). The flux calibration may be less reliable in these spectral regions, and may possibly not be accurately reflected in the associated errors of the [O II] lines.

In summary, the agreement matrix shown in Figure 9 highlights the limitations associated with defining consistent diagnostics relying on observational data, subject to observational and data processing errors. With a total of $2636(2.5 \%)$ of uncertain galaxies, the $13 \mathcal{Z E}$ diagnostics are nevertheless in very good agreement. For specific purposes, for example, if reddening corrections are large and/or uncertain, working with only a subset of $\mathcal{Z} \mathcal{E}$ diagnostics not involving the [O II] lines could lead to an even better agreement of the combined $\mathcal{Z} \mathcal{E}$ classification.

\section{DISCUSSION}

\subsection{Comparison of $\mathcal{Z E}$ Diagnostics with the Standard Optical Line Ratio Diagnostics}

Having introduced 13 new composite line ratio diagnostics, and estimated the uncertainties associated with galaxy classification when combining each of these diagrams, it behooves us to investigate how these new diagnostics compare with the three standard optical line ratio diagnostic diagrams: $\log [\mathrm{N} \mathrm{II}] / \mathrm{H} \alpha$ versus $\log [\mathrm{O} \mathrm{III}] / \mathrm{H} \beta, \log [\mathrm{S} \mathrm{II}] / \mathrm{H} \alpha$ versus $\log [\mathrm{O} \mathrm{III}] / \mathrm{H} \beta$, and $\log [\mathrm{O}$ I $] / \mathrm{H} \alpha$ versus $\log [\mathrm{O}$ III $] / \mathrm{H} \beta$.

For clarity, in Figures 10-12, we have separated out into three distinct diagrams the H II-like (left), the AGN-like (right), and the uncertain (center) galaxies, as defined by our new $\mathcal{Z E}$ diagnostics (see Section 5.2). The advantage of our new classification scheme is that by combining multiple diagnostics, we can assign a probability for all points to be H II-like or not. This probability (i.e., the number of consistent diagnostics) is color-coded and shown in the middle panel for all objects with uncertain classification. The concept of associating a probability with a given classification is reminiscent of the MEx diagram of Juneau et al. (2011), although our respective methods differ fundamentally in practice. Juneau et al. (2011) rely on a prior sample of SDSS galaxies first classified via two of the standard line ratio diagnostic diagrams to quantify the inherent additional

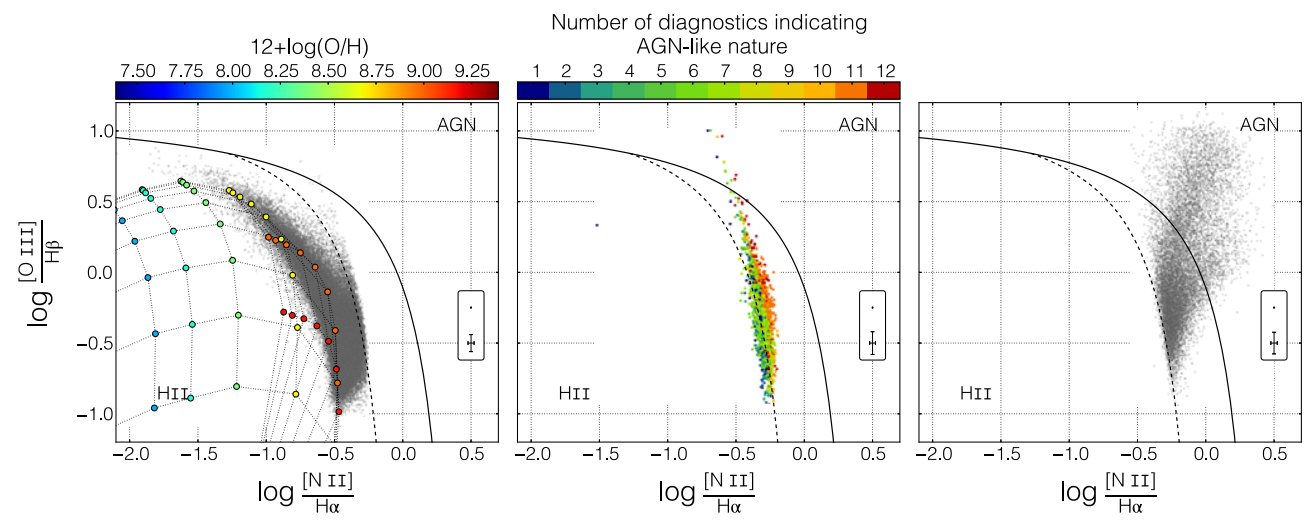

Figure 10. Location of the $\mathrm{H}$ II-like (left), AGN-like (right), and uncertain (middle) SDSS galaxies in the standard $\log [\mathrm{N}$ II $] / \mathrm{H} \alpha$ vs. $\log [\mathrm{O}$ III $] / \mathrm{H} \beta$ diagram. Uncertain galaxies are color-coded as a function of how many diagnostics indicate a non-H II-like nature. The diagnostic lines from Kewley et al. (2001a) and Kauffmann et al. (2003a) are marked with full and dashed lines, respectively. The different sectors (H II, AGN) are labeled following the nomenclature defined in Figure 4 of Kewley et al. (2006). We also show the MAPPINGS IV model grid for $\kappa=20$ in the left-hand diagram, where each grid point is color-coded as a function of $12+\log (\mathrm{O} / \mathrm{H})$. (A color version of this figure is available in the online journal.) 


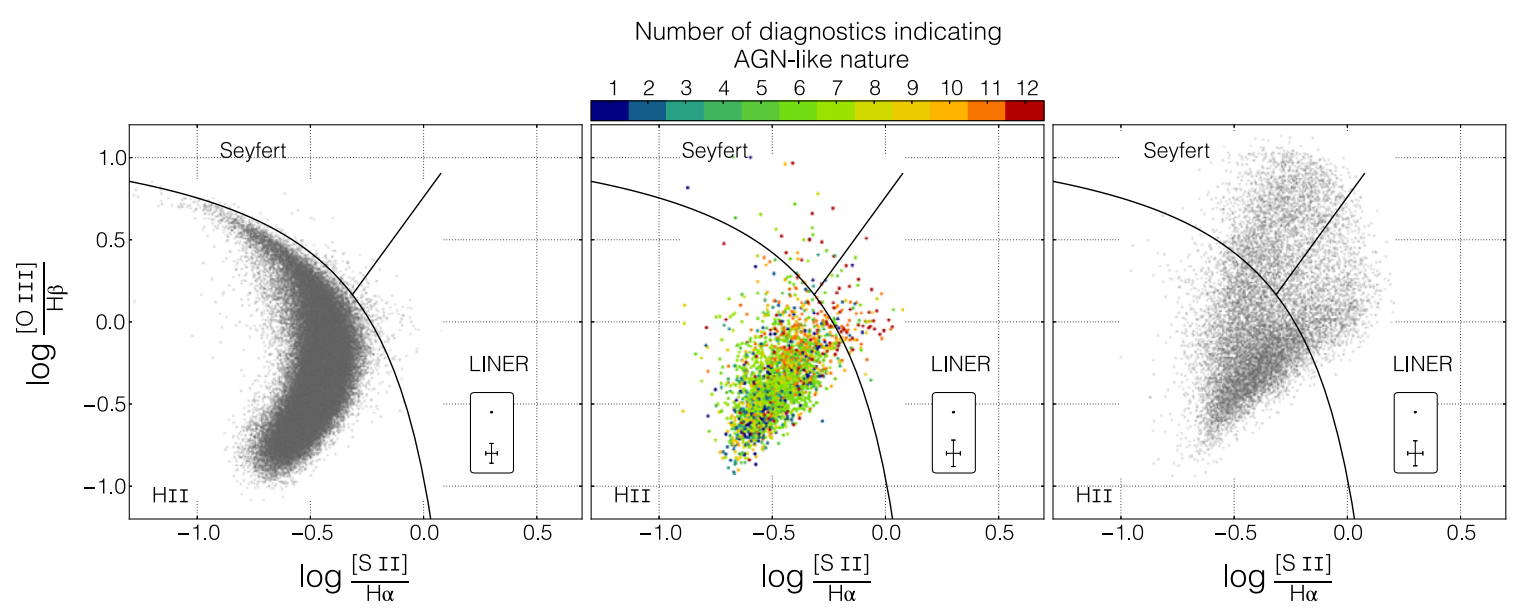

Figure 11. Same as Figure 10, but for the standard $\log [\mathrm{S} \mathrm{II}] / \mathrm{H} \alpha$ vs. $\log [\mathrm{O} \mathrm{III}] / \mathrm{H} \beta$ diagram. The maximum starburst line from Kewley et al. (2001a) and the Seyfert-LINER line from Kewley et al. (2006) are plotted accordingly.

(A color version of this figure is available in the online journal.)

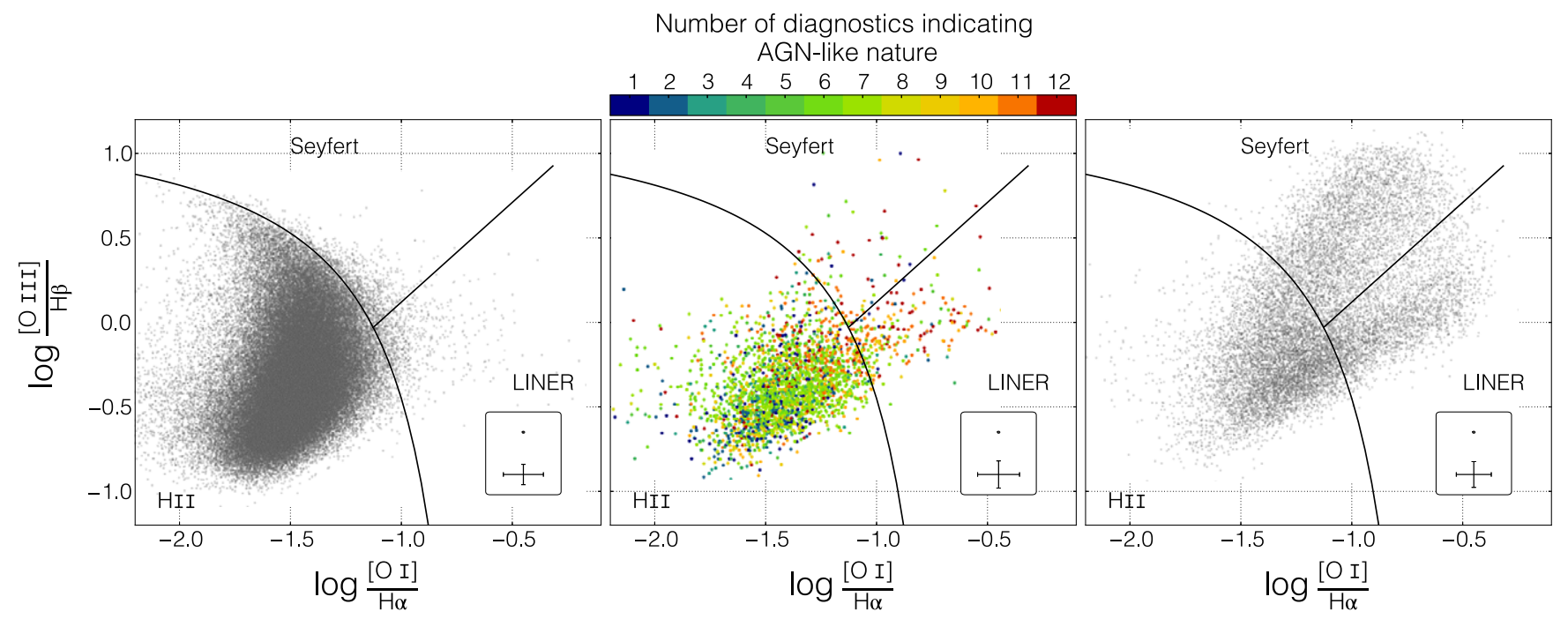

Figure 12. Same as Figure 10, but for the standard $\log [\mathrm{O} \mathrm{I}] / \mathrm{H} \alpha$ vs. $\log [\mathrm{O} I \mathrm{II}] / \mathrm{H} \beta$ diagram.

(A color version of this figure is available in the online journal.)

confusion between the different classes (star-forming, Seyferts, LINERs, composites) in the MEx diagram. On the other hand, with the $\mathcal{Z E}$ diagnostics, we derive a classification probability for the prior sample of SDSS galaxies itself, by combining 13 new and complementary line ratio diagnostic diagrams. In both cases, however, the need to quantify the certainty of a given classification is motivated by the fact that objects located near a given diagnostic line can have an inherently uncertain classification.

Clearly, our new composite diagnostics are concordant with the Kauffmann et al. (2003a) line in Figure 10, with only $1128(1.1 \%)$ galaxies classified with certainty by the $\mathcal{Z} \mathcal{E}$ diagnostics as being located on the wrong side of the Kauffmann et al. (2003a) diagnostic line. This agreement is in itself not surprising, since both approaches rely on an empirical fit of SDSS galaxies. However, we stress here that the $\mathcal{Z} \mathcal{E}$ diagnostics have all been defined before attempting any comparison with the standard diagnostic diagrams. The agreement between the $\mathcal{Z E}$ diagnostics and the Kauffmann et al. (2003a) diagnostic line is also a confirmation that, overall, objects defined as $\mathrm{H} \mathrm{II-}$ like based on the $\mathcal{Z E}$ diagnostics are indeed similar to star- forming galaxies classified using the standard line ratio diagram $\log [\mathrm{N}$ II $] / \mathrm{H} \alpha$ versus $\log [\mathrm{O}$ III $] / \mathrm{H} \beta$. On the other hand, the AGNlike objects (as defined by the $\mathcal{Z E}$ diagnostics) can be associated with the "traditional" Seyfert, LINER, and composite classes.

From the distribution of uncertain galaxies in the middle panel of Figure 10, it is clear that the $\mathcal{Z E}$ diagnostics are more consistent with the Kauffmann et al. (2003a) diagnostic toward the locus of the SDSS galaxies. For $\log [\mathrm{O}$ III $] / \mathrm{H} \beta \gtrsim 0$, the distribution of uncertain galaxies, which effectively trace the location of the boundary between H II-like versus AGNlike objects as defined by the $\mathcal{Z E}$ diagnostics, is not following the original diagnostic lines. The scarcity of galaxies present in this area make it difficult to objectively decide which set of diagnostics may be more appropriate, and further observations are required (for example, of very metal poor galaxies).

There is a smooth left to right gradient in the number of $\mathcal{Z E}$ diagnostics classifying the object as AGN-like through the zone occupied by the objects with uncertain classification in the $\log [\mathrm{N}$ II $] / \mathrm{H} \alpha$ versus $\log [\mathrm{O}$ III $] / \mathrm{H} \beta$ diagram. This gradient suggests that galaxies with at least $85 \%$ agreement between the different $\mathcal{Z E}$ diagnostics can be classified accurately. In addition, 
Table 6

Number of Uncertain Classifications Resulting from Different Combinations of Standard Optical Line Ratio Diagnostics and $\mathcal{Z} \mathcal{E}$ Diagnostics

\begin{tabular}{lccc}
\hline \hline Diagnostics & H II-like & AGN-like & Uncertain \\
\hline $\mathcal{Z} \mathcal{E}$ & 88918 & 13516 & 2636 \\
& $(84.6 \%)$ & $(12.9 \%)$ & $(2.5 \%)$ \\
{$[\mathrm{N} \mathrm{II}] / \mathrm{H} \alpha ;[\mathrm{O}$ III $] / \mathrm{H} \beta$} & 88933 & 16137 & $\ldots$ \\
{$[\mathrm{N} \mathrm{II}] / \mathrm{H} \alpha ;[\mathrm{O}$ III $] / \mathrm{H} \beta$} & $(84.6 \%)$ & $(15.4 \%)$ & \\
{$[\mathrm{S} \mathrm{II}] / \mathrm{H} \alpha ;[\mathrm{O}$ III $] / \mathrm{H} \beta$} & 88729 & 5377 & 10964 \\
{$[\mathrm{~N}$ II $] / \mathrm{H} \alpha ;[\mathrm{O}$ III $] / \mathrm{H} \beta$} & $(84.5 \%)$ & $(5.1 \%)$ & $(10.4 \%)$ \\
{$[\mathrm{S} \mathrm{II}] / \mathrm{H} \alpha ;[\mathrm{O}$ III $] / \mathrm{H} \beta$} & 86942 & & 12920 \\
{$[\mathrm{O}$ I $] / \mathrm{H} \alpha ;[\mathrm{O}$ III $] / \mathrm{H} \beta$} & $(82.7 \%)$ & $(5.0 \%)$ & $(12.3 \%)$ \\
\hline
\end{tabular}

we also note that if the galaxies with uncertain classifications mostly cluster around the starburst line from Kauffmann et al. (2003a), many galaxies with "certain" classification from the $\mathcal{Z} \mathcal{E}$ diagnostics also lie very close to the line.

The other two standard optical line ratio diagnostic diagrams $(\log [\mathrm{S} \mathrm{II}] / \mathrm{H} \alpha$ versus $\log [\mathrm{O}$ III $] / \mathrm{H} \beta$ and $\log [\mathrm{O}$ I $] / \mathrm{H} \alpha$ versus $\log [\mathrm{O} \mathrm{III}] / \mathrm{H} \beta$ ) are known to be less efficient at separating star-forming objects from AGNs. The reason, clearly visible in Figures 11 and 12, is that the Seyfert/LINER branches extend deeply in the star-forming regions (Kewley et al. 2006; Yuan et al. 2010). Consequently, these diagrams are in practice rarely used to identify star-forming galaxies, with the diagnostics solely relying on the $\log [\mathrm{N}$ II $] / \mathrm{H} \alpha$ versus $\log \left[\mathrm{O}_{\mathrm{III}}\right] / \mathrm{H} \beta$ diagram. For completeness, we list in Table 6 the percentage of "standard" uncertain galaxies that would result from different combinations of the three standard line ratio diagrams. To that end, we follow the criteria listed in Kewley et al. (2006), which we repeat here for clarity: "standard" star-forming galaxies lie below and to the left-hand side of the Kauffmann et al. (2003a) diagnostic in the $\log [\mathrm{N} \mathrm{II}] / \mathrm{H} \alpha$ versus $\log [\mathrm{O} \mathrm{III}] / \mathrm{H} \beta$ diagram, and below and to the left-hand side of the Kewley et al. (2001a) diagnostics in the $\log [\mathrm{S} \mathrm{II}] / \mathrm{H} \alpha$ versus $\log [\mathrm{O} \mathrm{III}] / \mathrm{H} \beta$ and $\log \left[\mathrm{O}_{\mathrm{I}}\right] / \mathrm{H} \alpha$ versus $\log \left[\mathrm{O}_{\mathrm{III}}\right] / \mathrm{H} \beta$ diagrams. For the sake of comparison with our $\mathcal{Z} \mathcal{E}$ diagnostics, we do not distinguish between composites, Seyfert, and LINERS, so that "standard" AGN-like objects lie above and to the right-hand side of the Kauffmann et al. (2003a) diagnostic in the $\log [\mathrm{N}$ II $] / \mathrm{H} \alpha$ versus $\log [\mathrm{O} \mathrm{III}] / \mathrm{H} \beta$ diagram, and above and to the right-hand side of the Kewley et al. (2001a) diagnostics in the $\log [\mathrm{S} \mathrm{II}] /$ $\mathrm{H} \alpha$ versus $\log [\mathrm{O} \mathrm{III}] / \mathrm{H} \beta$ and $\log \left[\mathrm{O}_{\mathrm{I}}\right] / \mathrm{H} \alpha$ versus $\log \left[\mathrm{O}_{\mathrm{III}}\right] / \mathrm{H} \beta$ diagrams. In that scheme, "standard" uncertain galaxies are those classified as star-forming in some diagrams, and AGN-like in others.

We did not apply any S/N cut on the [O I] line to obtain the standard classification. Indeed, the data presented in Table 6 are not intended as a mean of evaluating different classification strategies, but rather to provide a simple comparison of the size of the population of uncertain objects resulting from the combination of different line ratio diagnostics. As discussed previously, the $\mathcal{Z} \mathcal{E}$ diagnostics are very consistent with the standard $[\mathrm{N} \mathrm{II}] / \mathrm{H} \alpha$ versus $[\mathrm{O} \mathrm{III}] / \mathrm{H} \beta$ diagram alone. The number of galaxies classified as $\mathrm{H}$ II-like is very comparable $(0.1 \%$ change $)$ when combining both $\log \left[\mathrm{N}_{\mathrm{II}}\right] / \mathrm{H} \alpha$ versus $\log \left[\mathrm{O}_{\mathrm{III}}\right] / \mathrm{H} \beta$ and $\log \left[\mathrm{S}_{\mathrm{II}}\right] / \mathrm{H} \alpha$ versus $\log [\mathrm{O} \mathrm{III}] / \mathrm{H} \beta$, as most of the "standard" uncertain galaxies are in this case composed of AGN-like objects. Comparatively, combining all three standard optical line ratios does impact the number of galaxies classified as $\mathrm{H}$ IIlike by $\sim 2 \%$. The large amount of uncertain galaxies $(\sim 10 \%)$ resulting from the combination of different standard line ratio diagnostics is a direct consequence of the AGN branch extending deeply into the $\mathrm{HII}$-like region in the diagrams involving $\left[\mathrm{S}\right.$ II] and $\left[\mathrm{O}_{\mathrm{I}}\right]$. The number of uncertain galaxies associated with the more numerous $\mathcal{Z} \mathcal{E}$ diagnostics remains comparatively small $(2.5 \%)$ by design, as each diagnostic is chosen to cleanly separate the star-forming and AGN branches of SDSS galaxies.

As already discussed in Section 5.3, one disadvantage of the $\mathcal{Z} \mathcal{E}$ diagnostics is that they involve line ratios that are sensitive to reddening corrections. Comparatively, the standard [N II] $/ \mathrm{H} \alpha$ versus $[\mathrm{O} \mathrm{III}] / \mathrm{H} \beta$ is much more immune to this source of errors. We have mentioned in Section 5.3 the possibility of working with a subset of six $\mathcal{Z} \mathcal{E}$ diagnostics much less prone to reddening errors. However, the complete set of $\mathcal{Z} \mathcal{E}$ diagnostics might also act as a way of identifying possible reddening correction issues in a given data set, which would result in comparatively large numbers of uncertain $\mathcal{Z} \mathcal{E}$ classifications.

\subsection{ZE Diagrams for High Redshift Objects}

The limited number of atmospheric windows, and the stretching of the spectrum with redshift, makes the observation of the full set of emission lines of high-redshift galaxies difficult. At intermediate redshift $(0.5<z<1.0)$, the [S II], [N II], and $\mathrm{H} \alpha$ lines are redshifted in the near-infrared, while the [O II], [O III], and $\mathrm{H} \beta$ lines are still in the optical range. This observational constraint has motivated several hybrid alternatives to the standard line ratio diagnostic diagrams that replace the (inaccessible) redder line ratio with another observable one, such as $\log [\mathrm{O}$ II] $/ \mathrm{H} \beta$ (the "blue diagram"; see Lamareille et al. 2004; Lamareille 2010), the $U-B$ rest-frame color of the galaxy (the CEx diagram; Yan et al. 2011), or the galaxy's stellar mass (the MEx diagram; Juneau et al. 2011, 2014). Other propositions, such as the DEW diagram (Stasińska et al. 2006; Marocco et al. 2011) or the TBT diagram (Trouille et al. 2011), replaced both line ratios of the original diagnostics in favor of $D_{n}(4000)$ versus $\max (\mathrm{EW}[\mathrm{O} \mathrm{II}], \mathrm{EW}[\mathrm{Ne} \mathrm{III}])$ or the rest frame $g-z$ color versus $\log [\mathrm{Ne}$ III $] /[\mathrm{O}$ II $]$, respectively. In the case of $\mathcal{Z} \mathcal{E}$ diagnostics, the complete set relies on the measurement of (at least) one "red" line for the Category I (abundance-sensitive) line ratio, and at least one "blue" line for the Category II ( $q$-sensitive) line ratio (see Table 2). Hence, the $\mathcal{Z} \mathcal{E}$ diagnostics are subject to the same limitation as the original line ratio diagnostics for intermediate redshift objects.

Spectroscopic studies of galaxies at redshifts $1.0<z<1.7$ in the infrared miss observing the $[\mathrm{O}$ II $] \lambda 3727+\lambda 3729$ lines. While this fact rules out direct access to a certain number of line ratio diagrams, of the $13 \mathcal{Z E}$ diagnostics introduced in this article, six do not rely on [O II] (see Table 4 and Figure 5). Thus, while the full set is not available, the remainder should still be useful to ensure that a reliable classification can be made for high redshift objects. We stress that although the $\mathcal{Z} \mathcal{E}$ diagrams can be used, modeling of the evolution of metallicity and interstellar medium conditions is essential for understanding the evolution of $\mathcal{Z} \mathcal{E}$ diagnostics with redshift. The $\mathcal{Z} \mathcal{E}$ diagnostic lines are in fact subject to the same uncertainty as the standard diagnostic lines of Kewley et al. (2001a), Kauffmann et al. (2003a) and Kewley et al. (2006) at high redshifts (e.g., Liu et al. 2008; Brinchmann et al. 2008; Trump et al. 2013; Kewley et al. 2013a, 2013b; Juneau et al. 2014). 


\section{ALTERNATIVE APPLICATIONS FOR THE $\mathcal{Z} \mathcal{Q}$ 3D LINE RATIO DIAGRAMS}

In Sections 5 and 6, we used the $\mathcal{Z} \mathcal{Q} \mathcal{E}$ 3D optical line ratio diagrams to devise 13 new diagnostic diagrams that can separate H II-like and AGN-like objects in a consistent and robust way. A second application for $\mathcal{Z} \mathcal{Q E}$ diagrams is to perform a similar analysis specifically targeting the different classes of the AGN family: Seyfert, LINERs, and composites (which belong to the AGN-like group defined by the $\mathcal{Z} \mathcal{E}$ diagnostics). The complex structure of the AGN branch is visible in the interactive $\mathcal{Z} \mathcal{Q} \mathcal{E}_{\text {adg }}$ diagram in Figure 1. Other $\mathcal{Z} \mathcal{Q E}$ diagrams, for example, those involving the [O II] line, can provide an even cleaner separation between these different substructures. Hence, $\mathcal{Z} \mathcal{Q E}$ diagrams appear a useful tool for finding new view points on the AGN galaxies (in the multi-dimensional line ratio space), and to gain insight into the underlying physics by comparing these with theoretical models. This analysis, outside the scope of this article, will be the subject of a future publication.

As highlighted in Section 6.2, observational limitations for intermediate redshift galaxies can hinder the use of the $\mathcal{Z E}$ diagnostics. One solution to this limitation would be the creation of a series of hybrid $\mathcal{Z} \mathcal{Q E}$ diagrams, in which the inaccessible lines are replaced by alternative observables, similar to the MEx diagram (Juneau et al. 2011) and other similar 2D propositions. Depending on the data available, one could decide to either replace the "red" lines redshifted outside the optical region, or the "blue" lines not redshifted enough in the near-IR region. Similar to the approach applied in this article for the creation of the $\mathcal{Z} \mathcal{E}$ diagrams, the interactive inspection of a hybrid $\mathcal{Z} \mathcal{Q E}$ diagram might allow for the identification of specific points of view reducing the inherent confusion between the different classes of objects associated with the replacement of a line ratio with another observable - a common issue for all the "hybrid" 2D diagnostics mentioned in Section 6.2.

\section{SUMMARY}

In this article, we have demonstrated the utility of 3D line ratio diagrams for the classification of galaxies. Unlike standard (and historical) line ratio diagnostics in two dimensions, these new (interactive) diagrams allow for a better understanding of the spatial distribution of galaxies in the multi-dimensional line ratio space. Such diagrams are especially powerful when combing three different line ratios sensitive to (1) the gas-phase oxygen abundance $12+\log (\mathrm{O} / \mathrm{H})$, (2) the ionization parameter, $q$, and (3) the excitation mechanism. The key advantage of these 3D line ratio diagrams is to allow the identification of specific points of view of interest in the spatial distribution of galaxies.

In particular, we identified line-ratio triplets which, when projected onto a specific plane, compact the theoretical MAPPINGS $I V$ photoionization grids so that they are almost completely degenerate along the ionization parameter direction; in these triplets, the different chemical abundances of $\mathrm{H}$ II regions fall along a well-defined curve. From these specific viewpoints, we introduced 13 new composite line ratio diagnostics diagrams, the $\mathcal{Z} \mathcal{E}$ diagnostic diagrams, which enable us to efficiently separate $\mathrm{H}$ II-like objects from galaxies excited by AGNs independently of the standard optical line ratio diagrams.

We proved that this new set of composite line ratios is very consistent with the standard $\log [\mathrm{N}$ II $] / \mathrm{H} \alpha$ versus $\log \left[\mathrm{O}_{\mathrm{III}}\right] /$ $\mathrm{H} \beta$ diagnostic diagram, and especially with the Kauffmann et al. (2003a) diagnostic line. In other words, we have confirmed independently the known ability of the $\log [\mathrm{N}$ II $] / \mathrm{H} \alpha$ ver- sus $\log \left[\mathrm{O}_{\mathrm{III}}\right] / \mathrm{H} \beta$ diagram to efficiently separate star-forming galaxies from AGNs. The $\mathcal{Z} \mathcal{E}$ diagnostics also have the distinct advantage of attributing a probability for measurements to belong to the $\mathrm{H}$ iI-like class (or not) for galaxies close to the diagnostic boundaries. For our sample of 105,070 SDSS galaxies, we find that $2.5 \%$ have an uncertain classification when combining the $13 \mathcal{Z} \mathcal{E}$ diagnostics. Among our 13 new diagrams, six do not rely on $[\mathrm{O}$ II $] \lambda 3727+\lambda 3729$. These specific diagnostics are highly consistent ( $\sim 90 \%$ ) with one another, less prone to reddening correction issues, and are also suitable for spectroscopic studies of high-redshift objects which may not have access to the $[\mathrm{O}$ II $]$ emission lines.

The notion of a multi-dimensional, interactive line ratio diagram opens a new way of looking at the classification of extragalactic sources based on their emission line characteristics. Galaxies form a complex structure in this multi-dimensional space, a structure that is intrinsically hard to understand with $2 \mathrm{D}$ diagrams. With interactive $3 \mathrm{D}$ diagrams, we identified a new and complimentary technique to look at line ratio diagrams. This technique will be of special use in understanding the physical difference between the Seyfert and LINER classes of objects, which will be explored in a future paper.

We thank David Nicholls for stimulating discussions, Bill Roberts and the IT team at the Research School of Astronomy and Astrophysics (RSAA) at the Australian National University (ANU) for their support installing and maintaining the PDF3DReportGen software on the school servers, and the anonymous referee for his/her constructive review which helped us improve this article.

F. P. A. Vogt acknowledges a Fulbright scholarship and further financial support from an Alex Rodgers Travelling scholarship from the RSAA at the ANU. F. P. A. Vogt is also grateful to the Department of Physics and Astronomy at Johns Hopkins University for hosting him during his Fulbright exchange. M. A. Dopita acknowledges the support of the Australian Research Council (ARC) through Discovery project DP130103925. This work was funded by the Deanship of Scientific Research (DSR), King Abdulaziz University, under grant No. (5-130/1433 HiCi). The authors, therefore, acknowledge the technical and financial support of KAU. Scharwächter acknowledges the European Research Council for the Advanced Grant Program Num. 267399-Momentum.

This research has made use of NASA's Astrophysics Data System. Funding for SDSS-III has been provided by the Alfred P. Sloan Foundation, the Participating Institutions, the National Science Foundation, and the U.S. Department of Energy Office of Science. The SDSS-III Web site is http://www.sdss3.org/. SDSS-III is managed by the Astrophysical Research Consortium for the Participating Institutions of the SDSS-III Collaboration including the University of Arizona, the Brazilian Participation Group, Brookhaven National Laboratory, Carnegie Mellon University, University of Florida, the French Participation Group, the German Participation Group, Harvard University, the Instituto de Astrofisica de Canarias, the Michigan State/Notre Dame/JINA Participation Group, Johns Hopkins University, Lawrence Berkeley National Laboratory, Max Planck Institute for Astrophysics, Max Planck Institute for Extraterrestrial Physics, New Mexico State University, New York University, Ohio State University, Pennsylvania State University, University of Portsmouth, Princeton University, the Spanish Participation Group, University of Tokyo, University of Utah, Vanderbilt University, University of Virginia, University of Washington, and Yale University. 

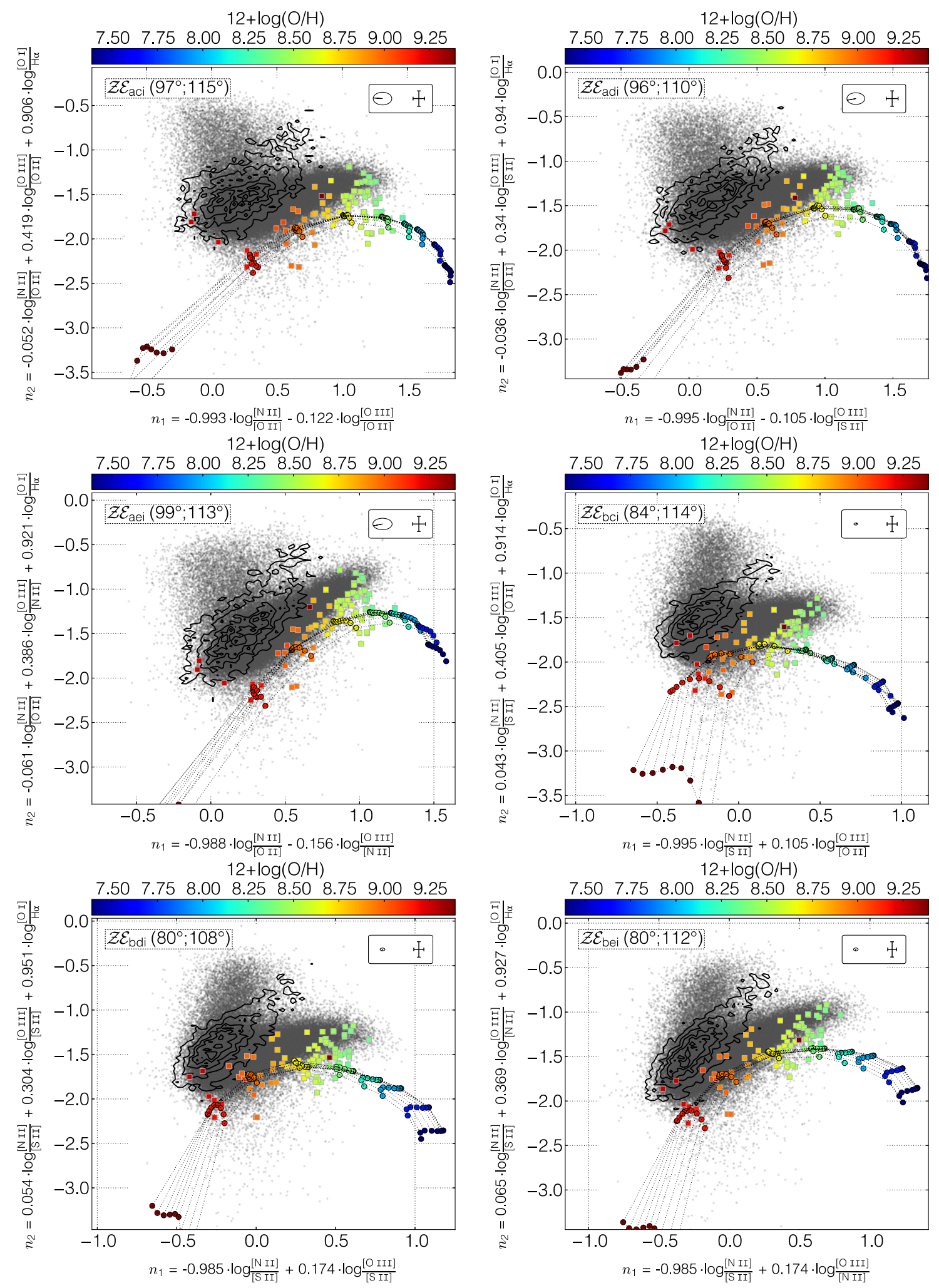

Figure 13. $\mathcal{Z E}$ diagrams for $\mathcal{Z} \mathcal{Q E}$ diagrams involving $\log [\mathrm{O} \mathrm{I}] / \mathrm{H} \alpha$ with no associated $\mathcal{Z E}$ diagnostic. The diagram name and associated values of $(\phi, \theta)$ are shown in the top left corner for completeness. H II-like and AGN-like SDSS galaxies are in gray. Uncertain galaxies (based on all $\mathcal{Z} \mathcal{E}$ diagnostics) are represented by density contours $(5 \%, 20 \%, 40 \%$ and $80 \%$ of the maximum density). The colored dots (connected by the dotted lines) correspond to the MAPPINGS IV models from Dopita et al. (2013). The van Zee et al. (1998) points are represented by small squares and the measurements from NGC 5427 are marked with small triangles. All measured $\mathrm{H}$ II regions are color-coded according to their oxygen abundance.

(A color version of this figure is available in the online journal.) 

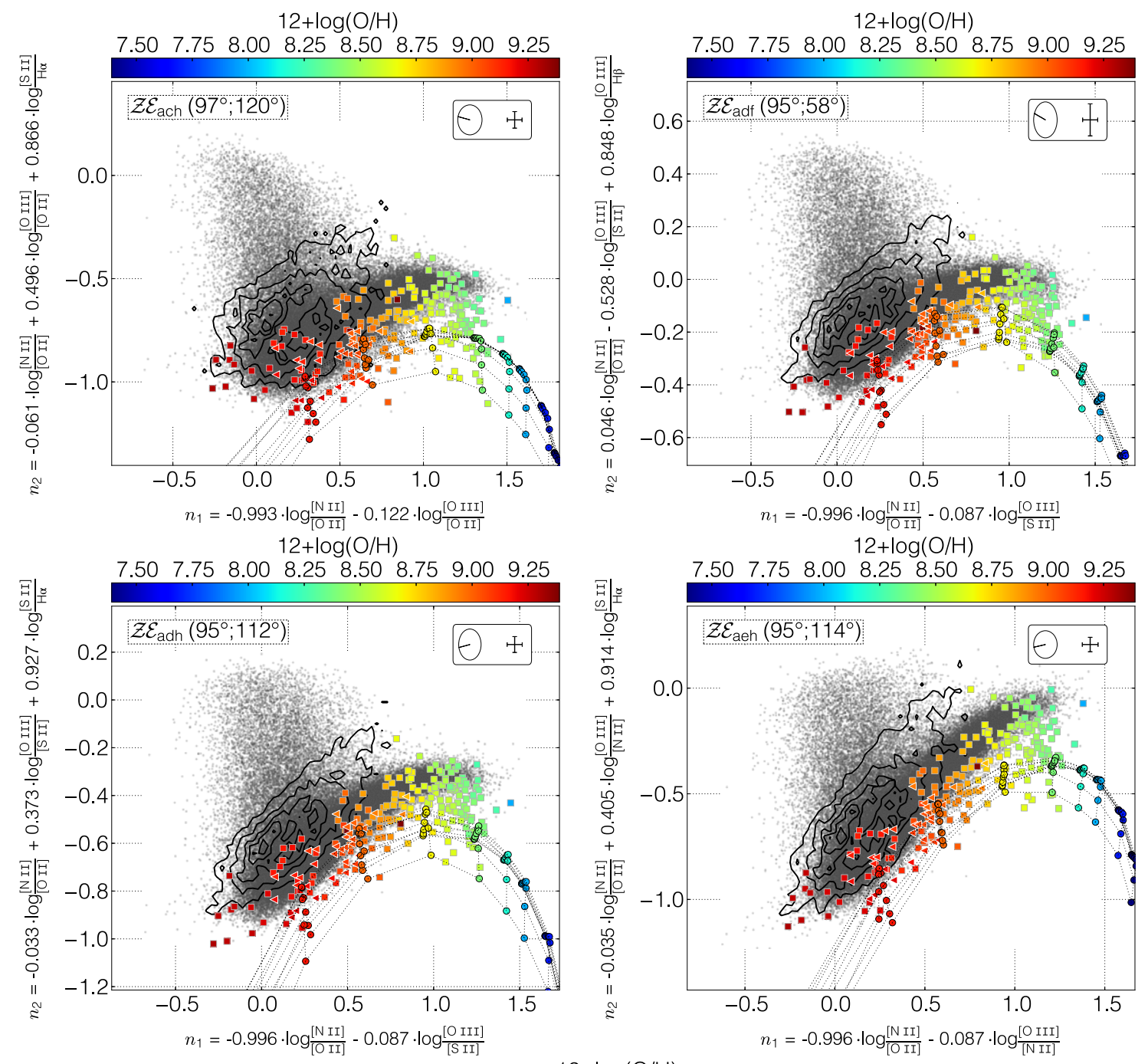

$12+\log (\mathrm{O} / \mathrm{H})$

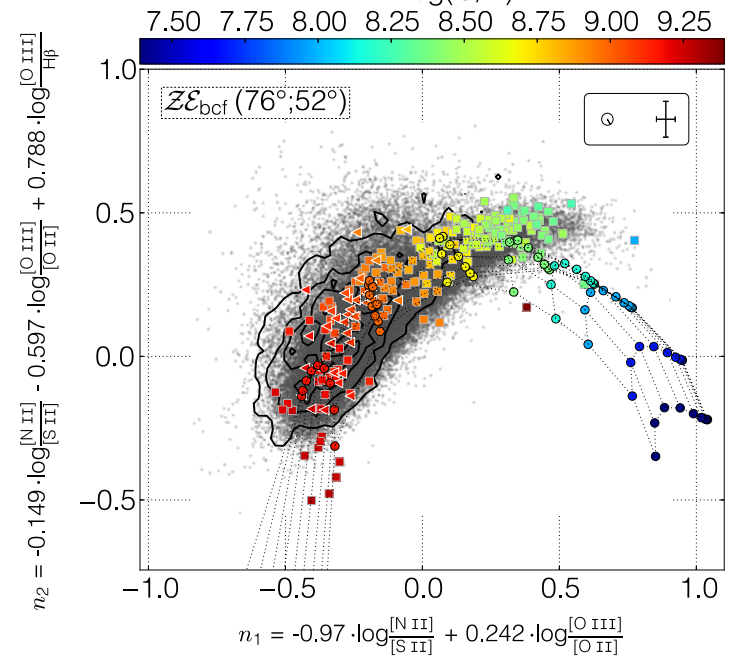

Figure 14. Same as Figure 13, but for $\mathcal{Z} \mathcal{Q E}$ diagnostics not involving $\log [\mathrm{O} \mathrm{I}] / \mathrm{H} \alpha$, and with no associated $\mathcal{Z} \mathcal{E}$ diagnostic.

(A color version of this figure is available in the online journal.)

\section{APPENDIX A}

\section{$\mathcal{Z} \mathcal{Q}$ DIAGRAMS WITH LARGER CONFUSION BETWEEN THE STARBURST AND AGN SEQUENCES}

Of the $24 \mathcal{Z} \mathcal{Q E}$ diagrams defined in Section 4, 11 have a comparatively higher confusion between the starburst and AGN branches of the cloud of points of SDSS galaxies, such that there is no viewpoint from which both branches can be clearly separated. In Figures 13 and 14, we show the $\mathcal{Z E}$ diagrams for each of the 11 "diagnostic-less" $\mathcal{Z} \mathcal{Q E}$ spaces that best collapse the starburst sequence onto itself. H II-like and AGN-like SDSS galaxies are in gray. Uncertain SDSS galaxies are once again 


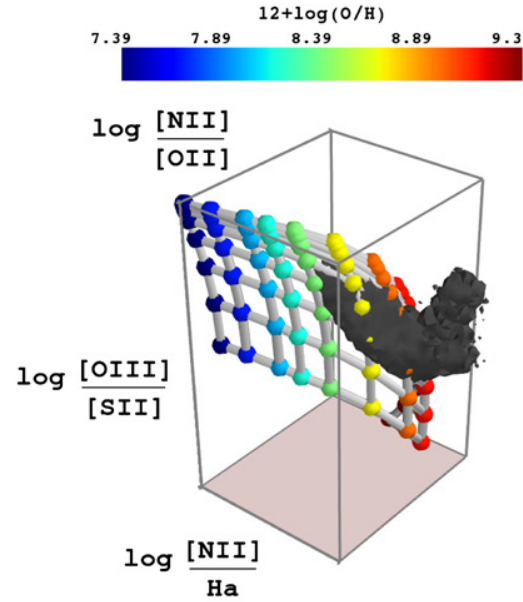

(a)

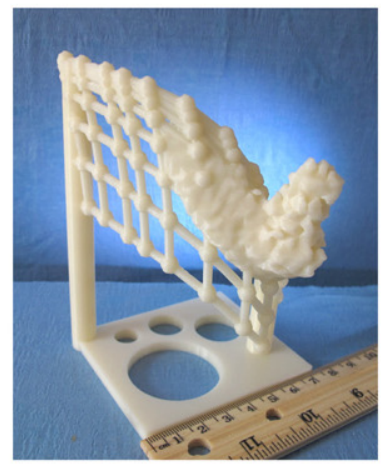

(b)

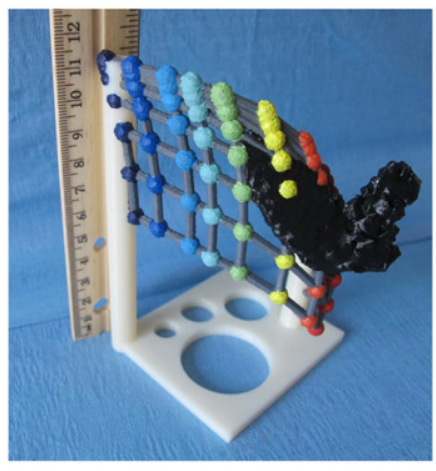

(c)

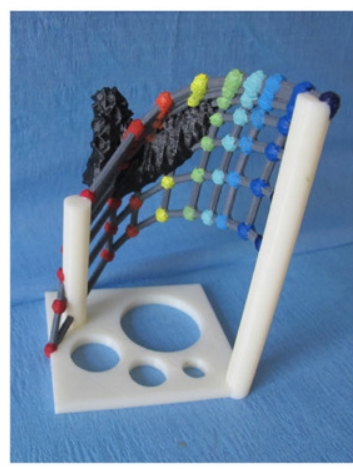

(d)

Figure 15. (a) $\mathcal{Z} \mathcal{Q} \mathcal{E}_{\text {adg }}$ as shown in Figure 1, but from a different point of view. SDSS galaxies are represented by a iso-density surface (black) instead of being shown individually as a cloud of point. (b) 3D printed model of $\mathcal{Z} \mathcal{Q} \mathcal{E}_{\text {adg }}$ using ABS plastic. The print direction was bottom to top, starting from the base plate. Two columns act as the support structure. The model size is approximatively $7 \mathrm{~cm} \times 7 \mathrm{~cm} \times 11 \mathrm{~cm}$. ((c) and (d)) Front and back views of the 3D printed model after applying a layer of acrylic paint manually to reproduce the oxygen abundance color scheme.

(A color version of this figure is available in the online journal.)

indicated via density contours. In all cases, both the distributions of the uncertain objects and of the starburst sequence are broad. Similarly, while the grid of $\mathrm{H}$ II photoionization models can be collapsed within $\sim 0.1 \mathrm{dex}$, the observational measurements of $\mathrm{H}$ II regions are more spread out. These diagrams may be of interest for different applications, such as the study of the AGN branch itself, although the points of view associated with the $\mathcal{Z E}$ diagrams in Figures 13 and 14 do not directly reveal the clear split between the different AGN classes in some of the associated $\mathcal{Z} \mathcal{Q E}$ diagrams.

\section{APPENDIX B}

\section{D PRINTING $\mathcal{Z} \mathcal{Q E}$ DIAGRAMS}

In an effort to explore new methods for sharing 3D structures such as $\mathcal{Z} \mathcal{Q E}$ diagrams, we give the interested reader the possibility to use 3D printing to construct a physical model of $\mathcal{Z} \mathcal{Q E}_{\text {adg }}$ (shown in Figure 1). The concept is similar to Steffen et al. (2014) and their 3D printable model of the Homunculus nebula around Eta Carina. A 3D printed model of $\mathcal{Z} \mathcal{Q} \mathcal{E}_{\text {adg }}$ is shown in Figure 15. The model is composed of the grid of MAPPINGS IV simulations, of an iso-density surface (black) tracing the position of SDSS galaxies, and of a support structure composed of two cylindrical columns and a base plate. The entire structure is defined in an .STL file available in the online journal. This file format ought to be compatible with most (if not all) of the 3D printers currently on the market. Having access to a monochromatic printer only, we have used acrylic paint to reproduce the color scheme tracing the oxygen abundance of MAPPINGS IV simulations.

The attached .STL file has been designed to be ready to print, and should not require any additional modifications before being sent to a 3D printer. While the presence of large gaps would benefit from the use of a dissolvable support material (and therefore a 3D printer with dual extrusion), the model is also compatible with less advanced, single extrusion devices.

The 3D model was first generated with the Mayavi2 module in Python, and saved as a .VRML/.WRL file. We manually add the external support structure using the freely available Blender software, save the entire structure to .OBJ format, and finally convert it to an .STL file with the freely available Meshlab software. We invite readers with practical questions regarding the $3 \mathrm{D}$ printing of the $\mathcal{Z} \mathcal{Q} \mathcal{E}_{\text {adg }}$ diagram, the structure design, or the file creation process to contact us directly.

\section{REFERENCES}

Abazajian, K. N., Adelman-McCarthy, J. K., Agüeros, M. A., et al. 2009, ApJS, 182,543

Adelman-McCarthy, J. K., Agüeros, M. A., Allam, S. S., et al. 2006, ApJS, 162,38

Aihara, H., Allende Prieto, C., An, D., et al. 2011a, ApJS, 193, 29

Aihara, H., Allende Prieto, C., An, D., et al. 2011b, ApJS, 195, 26

Allen, M. G., Groves, B. A., Dopita, M. A., Sutherland, R. S., \& Kewley, L. J. 2008, ApJS, 178, 20

Baldwin, J. A., Phillips, M. M., \& Terlevich, R. 1981, PASP, 93, 5

Barnes, D. G., \& Fluke, C. J. 2008, NewA, 13, 599

Bernardi, M., Sheth, R. K., Annis, J., et al. 2003, AJ, 125, 1866

Binette, L., Dopita, M. A., Dodorico, S., \& Benvenuti, P. 1982, A\&A, 115, 315

Binette, L., Dopita, M. A., \& Tuohy, I. R. 1985, ApJ, 297, 476

Brinchmann, J., Charlot, S., White, S. D. M., et al. 2004, MNRAS, 351, 1151

Brinchmann, J., Pettini, M., \& Charlot, S. 2008, MNRAS, 385, 769

Bruzual, G., \& Charlot, S. 2003, MNRAS, 344, 1000

Bruzual, G., \& Charlot, S. 2011, GALAXEV: Evolutionary Stellar Population Synthesis Models, Astrophysics Source Code Library

Buschkamp, P., Seifert, W., Polsterer, K., et al. 2012, Proc. SPIE, 8446, 84465

Calzetti, D., Armus, L., Bohlin, R. C., et al. 2000, ApJ, 533, 682

Charlot, S., \& Fall, S. M. 2000, ApJ, 539, 718

Croom, S. M., Lawrence, J. S., Bland-Hawthorn, J., et al. 2012, MNRAS, 421,872

Davies, R. L., Rich, J. A., Kewley, L. J., \& Dopita, M. A. 2014, MNRAS, 439, 3835

Djorgovski, S., \& Davis, M. 1987, ApJ, 313, 59

Dopita, M. A., Binette, L., \& Schwartz, R. D. 1982, ApJ, 261, 183

Dopita, M. A., Kewley, L. J., Heisler, C. A., \& Sutherland, R. S. 2000, ApJ, 542,224

Dopita, M. A., Rich, J., Vogt, F. P. A., et al. 2014a, Ap\&SS, 350, 741

Dopita, M. A., Scharwaechter, J., Shastri, P., et al. 2014b, A\&A, 566, A41

Dopita, M. A., Sutherland, R. S., Nicholls, D. C., Kewley, L. J., \& Vogt, F. P. A. 2013, ApJS, 208, 10

Dressler, A., Lynden-Bell, D., Burstein, D., et al. 1987, ApJ, 313, 42

Driver, S. P., Norberg, P., Baldry, I. K., et al. 2009, A\&G, 50, 050000

Eikenberry, S., Elston, R., Raines, S. N., et al. 2008, Proc. SPIE, 7014, 70140

Eisenstein, D. J., Weinberg, D. H., Agol, E., et al. 2011, AJ, 142, 72

Farage, C. L., McGregor, P. J., Dopita, M. A., \& Bicknell, G. V. 2010, ApJ, 724,267

Fischera, J., \& Dopita, M. 2005, ApJ, 619, 340 
Francis, P. J., \& Wills, B. J. 1999, in ASP Conf. Ser. 162, Quasars and Cosmology, ed. G. Ferland \& J. Baldwin (San Francisco, CA: ASP), 363

Groves, B., Brinchmann, J., \& Walcher, C. J. 2012, MNRAS, 419, 1402

Groves, B. A., Dopita, M. A., \& Sutherland, R. S. 2004, ApJS, 153, 9

Heckman, T. M. 1980, A\&A, 87, 152

Ho, L. C., Filippenko, A. V., \& Sargent, W. L. W. 1997, ApJS, 112, 315

Hunter, J. D. 2007, CSE, 9, 90

Jorgensen, I., Franx, M., \& Kjaergaard, P. 1996, MNRAS, 280, 167

Juneau, S., Bournaud, F., Charlot, S., et al. 2014, ApJ, 788, 88

Juneau, S., Dickinson, M., Alexander, D. M., \& Salim, S. 2011, ApJ, 736, 104

Kauffmann, G., Heckman, T. M., Tremonti, C., et al. 2003a, MNRAS, 346, 1055

Kauffmann, G., Heckman, T. M., White, S. D. M., et al. 2003b, MNRAS, 341,33

Kewley, L. J., Dopita, M. A., Leitherer, C., et al. 2013a, ApJ, 774, 100

Kewley, L. J., Dopita, M. A., Sutherland, R. S., Heisler, C. A., \& Trevena, J. 2001a, ApJ, 556, 12

Kewley, L. J., Groves, B., Kauffmann, G., \& Heckman, T. 2006, MNRAS, 372,961

Kewley, L. J., Heisler, C. A., Dopita, M. A., \& Lumsden, S. 2001b, ApJS, 132,37

Kewley, L. J., Jansen, R. A., \& Geller, M. J. 2005, PASP, 117, 227

Kewley, L. J., Maier, C., Yabe, K., et al. 2013b, ApJL, 774, L10

Kimura, M., Maihara, T., Iwamuro, F., et al. 2010, PASJ, 62, 1135

Lamareille, F. 2010, A\&A, 509, A53

Lamareille, F., Mouhcine, M., Contini, T., Lewis, I., \& Maddox, S. 2004, MNRAS, 350, 396

Levesque, E. M., Kewley, L. J., \& Larson, K. L. 2010, AJ, 139, 712

Liu, X., Shapley, A. E., Coil, A. L., Brinchmann, J., \& Ma, C.-P. 2008, ApJ, 678,758

Maragkoudakis, A., Zezas, A., Ashby, M. L. N., \& Willner, S. P. 2014, MNRAS, 441, 2296

Markwardt, C. B. 2009, in ASP Conf. Ser. 411, Astronomical Data Analysis Software and Systems XVIII, ed. D. A. Bohlender, D. Durand, \& P. Dowler (San Francisco, CA: ASP), 251

Marocco, J., Hache, E., \& Lamareille, F. 2011, A\&A, 531, A71

McLean, I. S., Steidel, C. C., Epps, H., et al. 2010, Proc. SPIE, 7735, 77351
McLeod, B. A., Fabricant, D., Geary, J., et al. 2004, Proc. SPIE, 5492, 1306 Nicholls, D. C., Dopita, M. A., \& Sutherland, R. S. 2012, ApJ, 752, 148 Nicholls, D. C., Dopita, M. A., Sutherland, R. S., Kewley, L. J., \& Palay, E. 2013, ApJS, 207, 21

Nicholls, D. C., Dopita, M. A., Sutherland, R. S., et al. 2014, ApJ, 786, 155

O’Donnell, J. E. 1994, ApJ, 422, 158

Osterbrock, D. E. 1989, Astrophysics of Gaseous Nebulae and Active Galactic Nuclei (Sausalito, CA: University Science Books)

Osterbrock, D. E., \& Pogge, R. W. 1985, ApJ, 297, 166

Ramachandran, P., \& Varoquaux, G. 2011, CSE, 13, 40

Rich, J. A., Kewley, L. J., \& Dopita, M. A. 2011, ApJ, 734, 87

Rich, J. A., Kewley, L. J., \& Dopita, M. A. 2014, ApJL, 781, L12

Rich, J. A., Torrey, P., Kewley, L. J., Dopita, M. A., \& Rupke, D. S. N. 2012, ApJ, 753,5

Sánchez, S. F., Kennicutt, R. C., Gil de Paz, A., et al. 2012, A\&A, 538, A8

Saulder, C., Mieske, S., Zeilinger, W. W., \& Chilingarian, I. 2013, A\&A, 557, A 21

Scharwächter, J., Dopita, M. A., Zuther, J., et al. 2011, AJ, 142, 43

Smee, S. A., Gunn, J. E., Uomoto, A., et al. 2013, AJ, 146, 32

Stasińska, G., Cid Fernandes, R., Mateus, A., Sodré, L., \& Asari, N. V. 2006, MNRAS, 371, 972

Steffen, W., Teodoro, M., Madura, T. I., et al. 2014, MNRAS, 442, 3316

Sutherland, R. S., \& Dopita, M. A. 1993, ApJS, 88, 253

Tremonti, C. A., Heckman, T. M., Kauffmann, G., et al. 2004, ApJ, 613, 898

Tresse, L., Rola, C., Hammer, F., et al. 1996, MNRAS, 281, 847

Trouille, L., Barger, A. J., \& Tremonti, C. 2011, ApJ, 742, 46

Trump, J. R., Konidaris, N. P., Barro, G., et al. 2013, ApJL, 763, L6

van Zee, L., Salzer, J. J., Haynes, M. P., O’Donoghue, A. A., \& Balonek, T. J. 1998, AJ, 116, 2805

Veilleux, S., \& Osterbrock, D. E. 1987, ApJS, 63, 295

Vogt, F., \& Wagner, A. Y. 2012, Ap\&SS, 337, 79

Vogt, F. P. A., Dopita, M. A., \& Kewley, L. J. 2013, ApJ, 768, 151

Vogt, F. P. A., \& Shingles, L. J. 2013, Ap\&SS, 347, 47

Wijesinghe, D. B., Hopkins, A. M., Sharp, R., et al. 2011, MNRAS, 410, 2291

Yan, R., Ho, L. C., Newman, J. A., et al. 2011, ApJ, 728, 38

York, D. G., Adelman, J., Anderson, J. E., Jr., et al. 2000, AJ, 120, 1579

Yuan, T.-T., Kewley, L. J., \& Sanders, D. B. 2010, ApJ, 709, 884 